\title{
Climatology of Medium-Scale Traveling Ionospheric Disturbances (MSTIDs) Observed with GPS Networks in the North African Region
}

Oluwadare T. Seun.

Institut fur Geodasie und Geoinformationstechnik, Technische Universitat Berlin, Str. des 17. Juni 135, 10623, Berlin, Germany. German Research Centre for Geosciences GFZ, Telegrafenberg, D-14473 Potsdam, Germany. oluwa@gfz-potsdam.de, temitopeseun@yahoo.co.uk

Norbert Jakowski.

German Aerospace Center (DLR), Institute of Communications and Navigation, Ionosphere Group, Kalkhorstweg 53, 17235 Neustrelitz, Germany

Norbert.Jakowski@dlr.de

Cesar E. Valladares.

W.B Hanson Center for Space Sciences, University of Texas at Dallas, USA Cesar.Valladares@utdallas.edu

Andrew Oke-Ovie Akala

Department of Physics, University of Lagos, Akoka, Yaba, Lagos, Nigeria aakala@unilag.edu.ng

Oladipo E. Abe

Physics Department, Federal University Oye-Ekiti, Nigeria

oladipo.abe@fuoye.edu.ng

Mahdi M. Alizadeh

Faculty of Geodesy and Geomatics Engineering, K.N. Toosi, University of Technology, Tehran, Iran.

Institut fur Geodasie und Geoinformationstechnik, Technische Universitat Berlin, Str. des 17. Juni 135, 10623, Berlin, Germany.

m.alizadeh@email.com

Harald Schuh.

Institut fur Geodasie und Geoinformationstechnik, Technische Universitat Berlin, Str. des 17. Juni 135, 10623, Berlin, Germany. German Research Centre for Geosciences GFZ, Telegrafenberg, D-14473 Potsdam, Germany. schuh@gfz-potsdam.de 


\title{
Climatology of Medium-Scale Traveling Ionospheric Disturbances (MSTIDs) Observed with GPS Networks in the North African Region
}

\author{
Oluwadare T. Seun ${ }^{1,2}$, Norbert Jakowski ${ }^{3}$, Cesar E. Valladares ${ }^{4}$, Andrew O. Akala ${ }^{5}$,
} Oladipo E. Abe ${ }^{6}$, Mahdi M. Alizadeh ${ }^{1,7}$, Harald Schuh ${ }^{1,2}$

\footnotetext{
${ }^{1}$ Institut fur Geodasie und Geoinformationstechnik, Technische Universitat Berlin, Str. des 17, 10623, Berlin, Germany

${ }^{2}$ German Research Centre for Geosciences GFZ, Telegrafenberg, D-14473 Potsdam, Germany

${ }^{3}$ German Aerospace Center (DLR), Institute of Communications and Navigation, Ionosphere Group, Neustrelitz, Germany

${ }^{4}$ W. B Hanson Center for Space Sciences, University of Texas at Dallas, USA

${ }^{5}$ Department of Physics, University of Lagos, Akoka, Yaba, Lagos, Nigeria

${ }^{6}$ Department of Physics, Federal University, Oye-Ekiti, Nigeria

${ }^{7}$ Faculty of Geodesy and Geomatics Engineering, K.N. Toosi, University of Technology,Tehran, Iran.
}

\section{Abstract}

We present for the first time the climatology of medium-scale traveling ionospheric disturbances (MSTIDs) by using Global Positioning System (GPS) receiver networks on geomagnetically quiet days (Kp $\leq 3)$ over the North African region during 2008-2016. The MSTIDs appear frequently as oscillating waves or wavelike structures in electron density induced by the passage of Atmospheric Gravity Waves (AGW) propagating through the neutral atmosphere and consequently, causing fluctuation in the ionospheric Total Electron Content (TEC). The TEC perturbations (dTEC) data are derived from dual frequency GPSmeasurements. We have statistically analyzed the MSTIDs characteristics, occurrence rate, seasonal behavior as well as the interannual dependence. The results show a local and seasonal dependence of nighttime and daytime MSTIDs. The propagation direction is predominantly towards the South (equatorward), MSTIDs event period is ( $12 \leq$ period $\leq 53$ mins), and dominant amplitude $(0.08 \leq \mathrm{amp} \leq$ $\sim 1.5$ TECU), with a propagation velocity higher at daytime than nighttime. The amplitudes of the MSTIDs increase with solar activity. The local MSTIDs Spatio-temporal heat reveals variability in disturbance occurrence time, but seems to be dominant within the hours of (Northwest: 1200-1600 LT) and (Northeast: 1000-1400 LT) in December solstice during daytime, and around (NW: 2100-0200 LT) and (NE: 19000200 LT) in June solstice, but get extended to March equinox during solar maximum (2014) during the nighttime. The time series of MSTIDs regional distribution map is also generated. Atmospheric gravity waves (AGW) seems to be responsible for the daytime MSTIDs occurrence.

Keywords: Medium scale traveling ionospheric disturbances, TEC perturbation, ionospheric irregularities, Atmospheric Gravity Waves, Total Electron Content 


\subsection{Introduction.}

Medium-scale traveling ionospheric disturbance (MSTID) is one of the major and frequent ionospheric irregularity phenomena at the F region Mid-latitude which may degrade positioning systems and it has been studied to have the ability to propagate over long distances (Frissell et al., 2014). MSTIDs could cause ionospheric biases and produce ionospheric disturbances that can degrade communication and navigation signals where the amplitude of MSTIDs is typical of tenth of a TECU ( 1 TECU $=10^{16}$ electrons $/ \mathrm{m}^{2}$ ) (Wanninger, 2004; Husin et al., 2011; Hernandes-Pajares et al., 2006), and have been thought to be generated by atmospheric gravity waves (AGWs) (Jonah et al., 2016). The AGWs have been proposed to be generated by the sunrise and sunset terminators (MacDougall et al., 2009a), also some studies have reported AGWs to be generated from convection activities (Tsuda et al., 2014) in the troposphere and stratosphere, and through some other meteorological processes (e.g., orography) (Jonah et al., 2016). AGWs are the most impactful waves that contribute to the dynamical nature of the upper atmosphere amongst many waves present in the atmosphere, and it is also an important energy transfer mechanism from troposphere into the stratosphere, mesosphere, and thermosphere (Jia Yue et al., 2019). AGW manifest as a wave-like perturbation of ionospheric plasma in the ionosphere (Hines, 1960), consequently causing fluctuations in Total Electron Content (TEC). The AGW propagation through the neutral atmosphere eventually gets above $50 \mathrm{~km}$ into the ionosphere and directly impacts it, causing ionospheric variability and seeds ionospheric irregularities (Azeem and Barlage, 2017) such as MSTIDs. These entire ionospheric frequent phenomena consequently cause fluctuations in Total Electron Content (TEC). The daytime MSTIDs event occurrence is majorly assumed to be caused by atmospheric gravity waves (AGW), (Hines, 1960; Jonah et al., 2016; Oinats et al., 2016; Figueiredo et al., 2018), while the nighttime is assumed to be caused by electrodynamical forces such as Perkins instability respectively (Perkins, 1973; Garcia et al., 2000; Tsugawa et al., 2007). The wavelike structure of MSTIDs is characterized by a wavelength, period and phase speed of 50 - 500 km, 12 - 60 mins and 50-400 m/s, respectively (Ogawa et al., 1987; Hocke and Schlegel, 1996; Grocott et al., 2013). Within the last six decades, a lot of MSTIDs studies have been carried out by various researchers around the globe using different instruments and techniques to understand this irregular ionospheric behavior. To mention a few amongst many are: Ogawa et al. (1987) who investigated MSTIDs occurrence frequency using the U.S. Navy Navigation Satellite System (NNSS) in polar region at a $1000 \mathrm{~km}$ altitude during the disturbed geomagnetic condition. The authors concluded that there was no increase in MSTIDs occurrence under disturbed condition. Hernández-Pajares et al. (2006, 2012), Kotake et al. (2007), Valladares and Hei, (2012), Jonah et al. (2016), Figueiredo et al. (2018), and Guanyi Chen et al. (2019) carried out independent research of MSTIDs using GNSS receiver network at difference location and they reported nearly the same results in terms of seasonal occurrences, but with slight differences in the propagation directions characteristics. Many studies have reported the regular and dynamic nature of the 
ionospheric TEC at different latitudes over the African region (Ouattara and Fleury, 2011; Ngwira et al., 2013; D'ujanga et al., 2016) but these earlier studies have only been confined to short-term time basis observation under limited solar activity. Besides, majority of the investigations reported to date have mostly emphasized the local or regional characteristics of ionospheric irregularities with main focus on scintillation. However, there is yet an important aspect of ionospheric irregularities which is yet to be reported on both local and regional scale over Africa. In recent years, with an improved study of long- term time series of characterization of ionospheric GPS-TEC under different geomagnetic conditions for daytime and nighttime, respectively, during 2009 - 2016 (Oluwadare et al., 2018), certain wave-like structures of ionospheric TEC were observed to be irregularities which vary in time and space. The characteristics of this irregular phenomenon are mostly associated with MSTIDs as described by ionospheric irregularity theories and experimental results from different authors who have reported MSTIDs observations from different regions e.g. North Asia, South America, North America, Europe, and Oceania around the globe except for the African region, and these have created a huge gap in comparison of interregional MSTIDs characteristics. Before now, MSTIDs study over the African region has not been reported, probably due to limitation of ionospheric data availability in the non-African region or none-access to the GPS data and other technical reasons. Hence, this study has considered further observing and investigating the dynamic nature of ionosphere and the occurrence of ionospheric irregularities with main focus on MSTIDs from GPS-TEC estimates. The study investigates the climatology of MSTIDs over the North African region for the first time during the geomagnetic quiet days (i.e. Kp $\leq 3$ ) for the period of nine years (2008-2016). The primary aim is to present the MSTIDs observations derived from estimated TEC perturbation (dTEC), characteristics and occurrence mechanism. We also show the perturbed temperature profile as tropospheric activities obtained from the Constellation Observing System for Meteorology, Ionosphere, and Climate (COSMIC) satellite which exhibits AGW signature and widely thought to be majorly due to the vertical propagation of AGW (Grant et al., 1998). For the first time, we present the nine years' climatology of MSTIDs observed by a GPS network over the North African region. The MSTIDs occurrence rate (OR) is estimated, its characteristics as well as the mechanisms are investigated. In addition, the regional distribution of MSTIDs occurrence map is presented and a daytime two-dimensional dTEC map for a case of MSTIDs was also presented. 


\subsection{Data and method}

MSTIDs have been observed and estimated during 2008-2016 using seven ground-based dual-frequency GPS receiver network stations majorly situated at Northern African, a mid-latitude region. The location of the stations is given in the table (1) below. The entire seven GPS network stations are shown in Fig. 1.

Table 1. GPS receiver stations showing geographical coordinate values and geomagnetic latitude values.

\begin{tabular}{llll}
\hline Station name & Geog. Lat & Geog. Lon & Geomag. Lat \\
\hline RABT & $33.99^{\circ} \mathrm{N}$ & $6.85^{\circ} \mathrm{W}$ & $37.4^{\circ} \mathrm{N}$ \\
\hline TETN & $35.56^{\circ} \mathrm{N}$ & $5.36^{\circ} \mathrm{W}$ & $38.6^{\circ} \mathrm{N}$ \\
\hline IFR1 & $33.51^{\circ} \mathrm{N}$ & $5.13^{\circ} \mathrm{W}$ & $36.7^{\circ} \mathrm{N}$ \\
\hline NOT1 & $36.88^{\circ} \mathrm{N}$ & $14.91^{\circ} \mathrm{E}$ & $36.5^{\circ} \mathrm{N}$ \\
\hline ALX2 & $31.20^{\circ} \mathrm{N}$ & $29.91^{\circ} \mathrm{E}$ & $28.5^{\circ} \mathrm{N}$ \\
\hline NICO & $35.14^{\circ} \mathrm{N}$ & $33.40^{\circ} \mathrm{E}$ & $31.8^{\circ} \mathrm{N}$ \\
\hline RAMO & $30.60^{\circ} \mathrm{N}$ & $34.76^{\circ} \mathrm{E}$ & $27.1^{\circ} \mathrm{N}$ \\
\hline
\end{tabular}

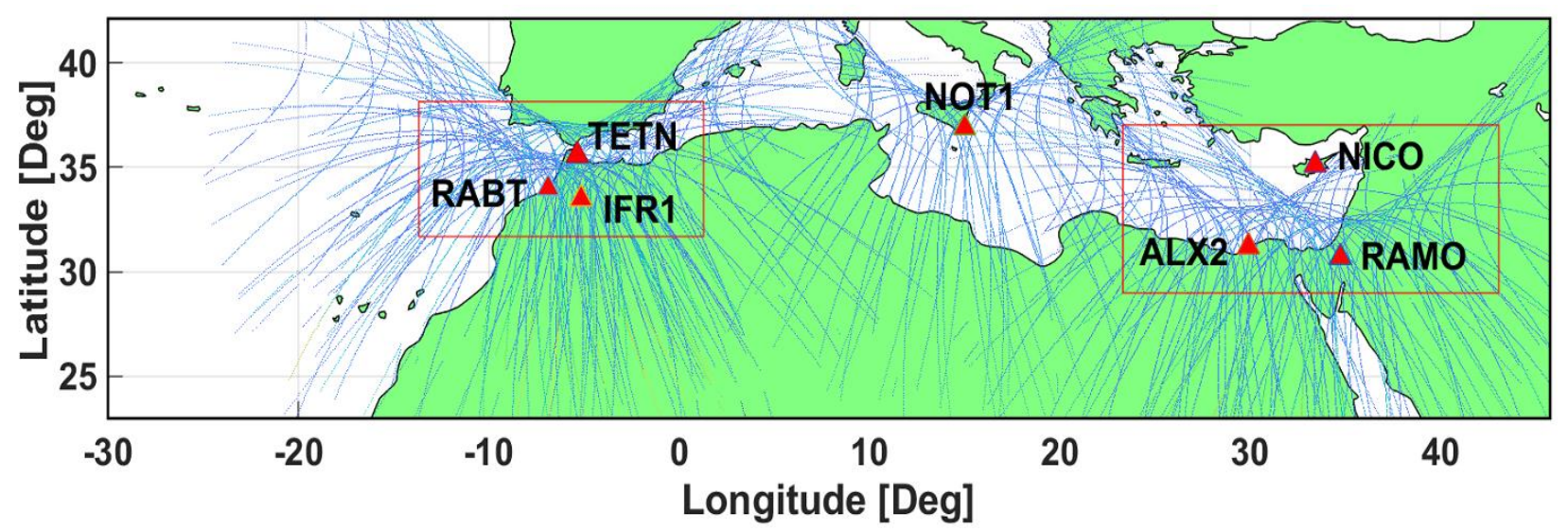

Fig. 1 Location of the GPS receiver stations (red triangles) used in this study with an elevation mask $\geq 35^{\circ}$. GPS geometric networks were formed by choosing minimum of three stations (enclosed in red box) to form new sub networks (N1: RABT-TETN-IFR1, N2: ALX2-NICO-RAMO)

The observation GPS data in Rinex format were obtained from the following FTP sites: ftp://dataout.unavco.org/pub/rinex/, ftp://igs.ign.fr/pub/igs/data/, http://www.afrefdata.org/ and ftp://www.stationgps.cea.com.eg/ALX2/, respectively. The GPS distribution is presented with red triangle with the corresponding IPP trajectory (blue color curve) of the GPS satellites (Fig. 1). To avoid water vapor scattering multipath effects and effect from the mapping function uncertainty from the data, an elevation cut-off angle greater than $35^{\circ}$ (Bagiya et al., 2009; Valladares and Hei, 2012) was adopted. 

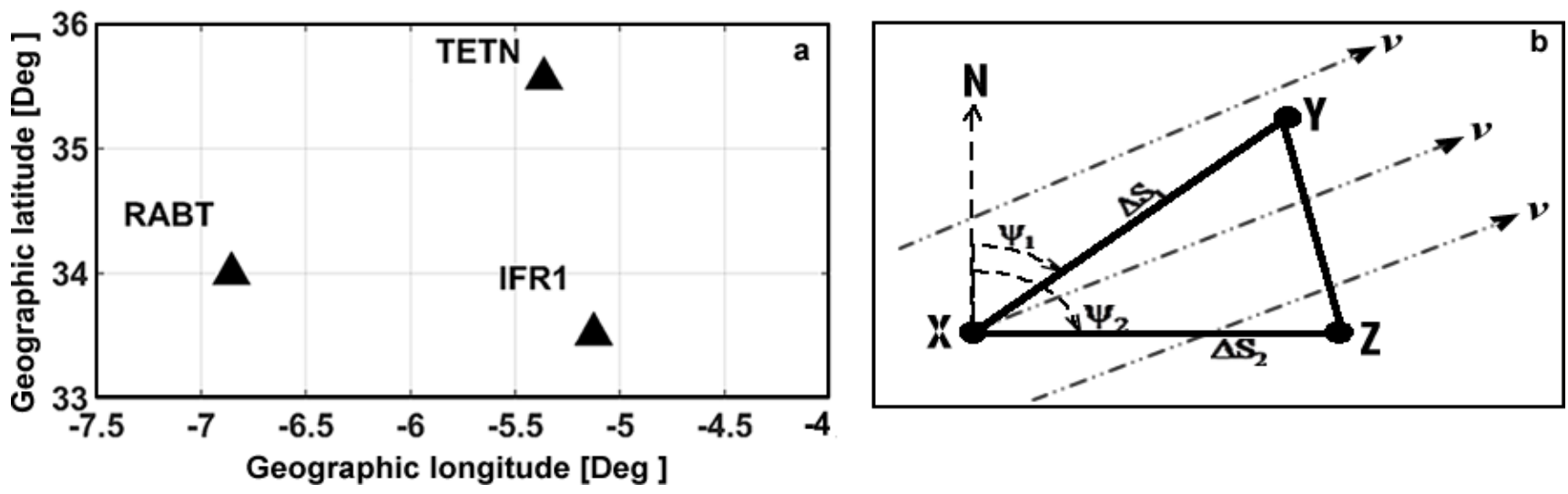

Fig. 2 (a) An example illustrating one of the sub-networks (RABT-TETN-IFR1) used in studying MSTIDs characteristics. (b) The configured network geometry for obtaining the MSTIDs propagation direction and velocity.

Also, the low geomagnetic activity conditions were considered using K planetary (Kp) index. The magnitude of $\mathrm{Kp}$ index $\leq 3$ was considered for low geomagnetic quiet conditions during the study period. The Kp index data were obtained from the GFZ German Research Centre for Geosciences, Indices of Global Geomagnetic Activity, Potsdam, Germany (ftp://ftp.gfz-potsdam.de/pub/home/obs/kp-ap). Days with $\mathrm{Kp} \leq 3$ during 2008-2016 were filtered out and used for this study. Furthermore, we made use of temperature profile data extracted from the Constellation Observing System for Meteorology, Ionosphere, and Climate (COSMIC) satellite. The COSMIC satellites are a low Earth orbit (LEO) satellite that provides both upper and lower atmospheric products for different altitude observations of temperature, the electron density of the ionosphere, pressure, refractivity, and water vapor. We observed and extracted perturbed temperature profile which may be due to the vertical propagation of AGWs from the COSMIC data (Grant et al., 1998). The COSMIC data are available at COSMIC Data Analysis and Archive Center (CDAAC) website (https://cdaac-www.cosmic.ucar.edu/cdaac/tar/rest.html).

\subsection{Estimation of Ionospheric GPS-TEC derived}

The ionospheric Total Electron Content (TEC) was derived from Global Positioning System (GPS) measurements. The GPS-TEC derived was used to capture the Medium-Scale Traveling Ionospheric Disturbances (MSTIDs). TEC was computed using dual frequencies GPS receivers in which the first carrier frequency $f_{1}$ is centered at $1575.42 \mathrm{MHz}$ and the second carrier frequency $f_{2}$ centered at $1227.60 \mathrm{MHz}$. Following Gao and Liu (2002), Carrano and Groves (2006), Zhao et al. (2009) and Abe et al. (2017), the code and carrier phase measurements obtained from GPS were used to compute the slant TEC (sTEC) along the signals path from the satellite to the receiver as follows:

$$
s \operatorname{TEC}_{p}=\frac{f_{1}^{2} f_{2}^{2}}{40.3\left(f_{1}^{2}-f_{2}^{2}\right)}\left[\left(P_{2}-P_{1}\right)-\left(B_{s}+B_{r}+\varepsilon_{p}\right)\right]
$$




$$
s T E C_{L}=\frac{f_{1}^{2} f_{2}^{2}}{40.3\left(f_{1}^{2}-f_{2}^{2}\right)}\left[\left(\lambda_{2} L_{2}-\lambda_{1} L_{1}\right)-\left(\lambda_{1} A_{1}+\lambda_{2} A_{2}+\varepsilon_{L}\right)\right]
$$

where $\mathrm{P}_{1}$ is the code-delay measurement on frequency $f_{1}(\mathrm{~m}), \mathrm{P}_{2}$ is the code-delay measurement on frequency $f_{2}(\mathrm{~m}), \mathrm{B}_{\mathrm{s}}$ is the satellite differential code biases $(\mathrm{m}), \mathrm{B}_{\mathrm{r}}$ is the receiver differential code biases $(\mathrm{m}), \mathrm{L}_{1}$ is the carrier phase measurement on frequency $f_{1}$ (cycles), $\mathrm{L}_{2}$ is the carrier phase measurement on frequency $f_{2}$ (cycles), $\mathrm{A}_{1}$ is the ambiguity integer measure on the carrier phase on $\mathrm{L}_{1}$ frequency (cycles), $\mathrm{A}_{2}$ is the ambiguity integer measure on the carrier phase on $\mathrm{L}_{2}$ frequency (cycles), $\varepsilon_{\mathrm{p}}$ is the noise within the frequency channel and multipath associated with the code-delay measurements $(\mathrm{m}), \varepsilon_{\mathrm{L}}$ is the noise and multipath associated with the carrier phase measurements (cycles), $\lambda_{1}$ and $\lambda_{2}$ are the wavelengths $(\mathrm{m})$ corresponding to $f_{1}$ and $f_{2}$, respectively. The sTECP obtained in equation (1) is much noisy due to the inbuilt noise in the frequency channel while the $\mathrm{STEC}_{\mathrm{L}}$ obtained in equation (2) is much ambiguous due to some cycle slips and many loss of lock (inability of the receiver to track the signals). The noisy but unambiguous STECP was used to level the STEC $_{L}$ to arrive at a logical sTEC that is neither noisy nor ambiguous. As STEC is dependent on the ray path geometry through the ionosphere, it is needful to calculate an equivalent vertical TEC (VTEC) value which is independent of the elevation of the ray path. Hence, the VTEC is obtained by taking the projection from the slant to vertical using a mapping function $\mathrm{M}(\theta)$ as contained in (Klobuchar, (1986); Mannucci et al. (1998); Ciraolo L, et al. (2006)),

$$
\begin{gathered}
V T E C=\operatorname{STEC} \times \mathrm{M}(\theta) \\
M(\theta)=\frac{V T E C}{S T E C}=\left[1-\left(\frac{R_{e} \cos (\theta)}{R_{e}+h_{\max }}\right)^{2}\right]^{\frac{1}{2}}
\end{gathered}
$$

where $\mathrm{Re}$ is the mean earth radius; $6371 \mathrm{~km}, \theta=$ elevation angle of the satellite in degrees, $\mathrm{h}_{\max }$ is the maximum height above the surface of the Earth, (Mannucci et al., 1993; Langley et al., 2002), $350 \mathrm{~km}$, has been taken to be $h_{\max }$ value, this is because at this height the ionosphere is assumed to be spatially uniform and simplified to be a thin layer, hence, this is considered as the height of maximum electron density at the F2 peak (Mannucci et al., 1998; Norsuzila et al., 2009). More details about VTEC estimation can be found in Mannucci et al. (1998) and Ciraolo L. et al (2006). The background trends of the TEC time series were obtained by using singular spectrum analysis (SSA) with sliding window duration of 60 mins and thereafter the output is subtracted from the original TEC time series resulting to TEC perturbation (dTEC), see equation (9) in the next section. 


\subsection{Fitting tool: Singular Spectrum Analysis (SSA)}

Different order of polynomial fittings as a band-pass technique to filter out diurnal variability and TEC perturbations associated with MSTIDs have been used in previous studies (Ding et al., 2004; Wang Min et al., 2007; Valladares and Hei, 2012; Jonah et al., 2016). However, most of these techniques have some limitations because the direction of the trend of the fitness line and degree of smoothness/resolution cannot be controlled due to imposition of predetermined function. This is the reason we adopted singular spectrum analysis (SSA) algorithm as a detrending tool for dTEC. Our choice of SSA (see equation 5-8) among other things is because it is a nonparametric spectral estimation method for time series which cannot be affected by the limitations described above and most importantly due to its ability to find trends of different degrees of resolutions. We use equations (5) to (8) to map the original one-dimensional TEC time series (i.e. FN) of length $\mathrm{N}$ into a multi-dimensional series of lagged vectors of size $\mathrm{L}$, where $\mathrm{N}$ is greater than two.

$$
\begin{aligned}
& \overbrace{f_{1}, f_{2}, \quad f_{3}, \ldots \ldots \ldots ., f_{L}}^{\text {window }}, f_{L+1}, \ldots \ldots \ldots, f_{N}, \text { implies } \boldsymbol{F}_{1}{ }^{T}=\left(f_{1}, \quad f_{2}, \quad f_{3}, \ldots \ldots \ldots . ., f_{L}\right) \\
& \text { window }
\end{aligned}
$$

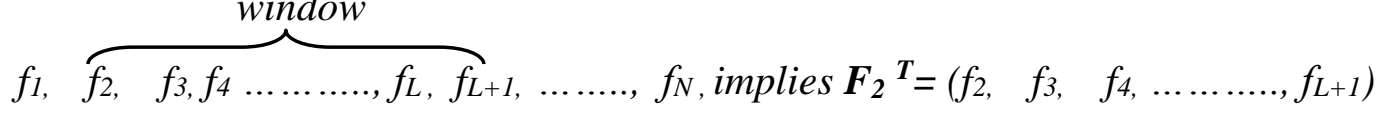

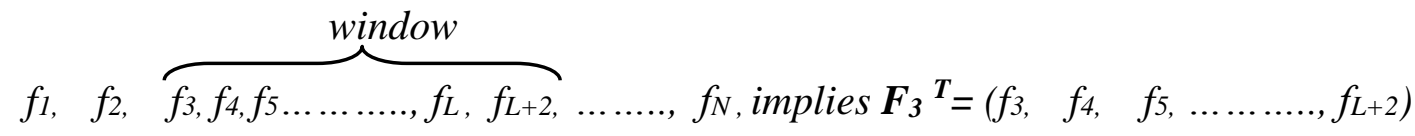

$$
\begin{aligned}
& F=\left[F_{1}, F_{2}, F_{3}, F_{4}, \ldots \ldots F_{K}\right]=\left(\begin{array}{cccc}
f_{1} & f_{2} & f_{3} & \ldots . . f_{K} \\
f_{2} & f_{3} & f_{4} & \ldots . . f_{K+1} \\
f_{3} & f_{4} & f_{5} & \ldots . . f_{K+2} \\
: & : & : & : \\
f_{L} & f_{L+1} & f_{L+2} & f_{N}
\end{array}\right),\left\{\begin{array}{l}
1<L<K \\
K=N-L+1
\end{array}\right.
\end{aligned}
$$

F implies TEC time series which formed a trajectory matrix $(\mathbf{F}), f_{\mathrm{i}}$ implies TEC values at each epoch of each PRN as time increases, and $f_{\mathrm{i}}$ must not be series of zeros, $i=1,23, \ldots . L$. Golyandina et al. (2001) provides further details about SSA.

\subsection{Estimation of TEC perturbation (dTEC) and MSTIDs event threshold}

An SSA fit is determined for each TEC time series (TECsSA-fit) of the corresponding satellite. The TEC perturbation (dTEC) is obtained by subtracting the TECssA-fit from the TEC estimate. 
The approach to obtain dTEC in equation (11) is known as detrending. We determine that an MSTID event is detected whenever the TEC perturbation (dTEC) points fall above the event threshold (ETH) value of 0.07 TECU (Husin et al., 2011). The choice of ETH value was based on computing the standard deviation of the TEC perturbation (dTEC) of all epochs per observed satellite (Warnant, 1998; Warnant and Pottiaux, 2000). We iterated the entire standard deviation process for several satellites for different days and then found an approximate value of the most dominant standard deviation value which we set as the ETH point value.
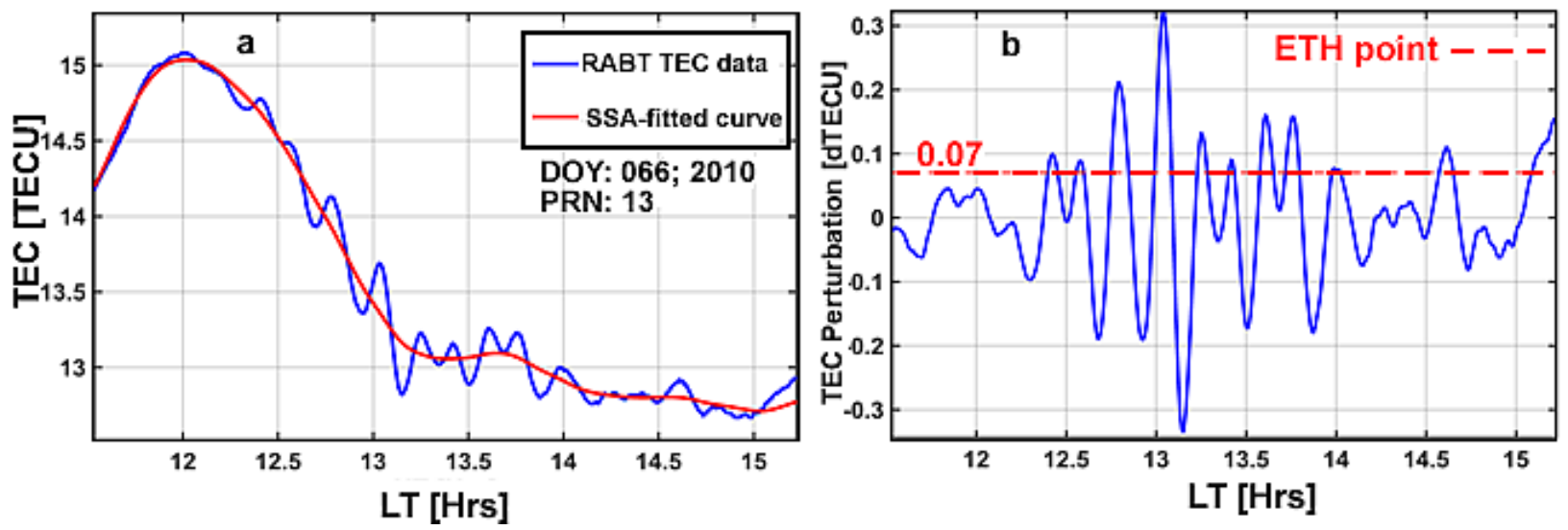

Fig. 3 (a) TEC time series in PRN 13 as observed at RABT GPS station exhibiting wave-like structures depicting to be MSTIDs. The red line fitted curve $\left(\right.$ TEC $_{\text {SSA-fit }}$ ) is the background trend while (b) is the corresponding detrended TEC time series known as dTEC.

\subsection{Determination of MSTIDs Characteristics}

In this study, we define MSTIDs as the dTEC that satisfy the following criteria: (1) the dTEC has as amplitude exceeding 0.07 TECU (1TECU $=10^{16}$ Electron $/ \mathrm{m}^{2}$ ) (Fig. 3b); (2) the horizontal wavelength is described as the distance between peak to peak of each wave event using visual assessment of dTEC signals and estimated to be less than $500 \mathrm{~km}$; (3) the dTEC series was transformed from the time domain to the frequency domain in order to determine the event dominant period using a Fast Fourier Transform (FFT) (Husin et al., 2011; Arikan et al., 2017) and the period is estimated to be less than 60 mins; (4) the propagation velocity does not exceed $450 \mathrm{~m} / \mathrm{sec}$. The geometry of calculating the MSTIDs propagation parameters is plotted in Fig. (2b) as an illustration in determining the azimuth and velocity. It must be noted that GPS receiver stations that are relatively close to each other are considered to form a sub-network (minimum of three stations) following the approach of Afraimovich et al. (1998); Hernández-Pajares et al. (2012); Valladares and Hei, (2012); and Habarulema et al. (2013a). Hence, we form a sub-network as seen in Fig.1, where the GPS receiver station RABT, TETN and IFR1 is represented by X, Y and Z, respectively in Fig 2(a-b). We assume that the TID's wavefront propagates along the Earth's spherical surface and crosses point positions $\mathrm{X}, \mathrm{Y}$ and $\mathrm{Z}$ with speed $\boldsymbol{v}$ and propagation azimuth ( $\phi)$. The azimuth is measured from the north $(\mathbf{N})$ towards the east along the horizon. The phase fronts propagation velocity satisfies the 
equations below (Ding et al., 2007).

$\mathrm{V} \Delta \mathrm{t}_{1}=\Delta \mathrm{S}_{1} \cos \left(\Phi-\psi_{1}\right), \mathrm{V} \Delta \mathrm{t}_{2}=\Delta \mathrm{S}_{2} \cos \left(\Phi-\psi_{2}\right)$

Where $\Delta \mathrm{t}_{1}$ and $\Delta \mathrm{t}_{2}$ are time delays for dTEC to move from point $\mathrm{X}$ to $\mathrm{Y}$ and $\mathrm{Z}$ respectively along the Earth spherical surface and computed using cross-correlation. $\Delta \mathrm{S}_{1}$ is the spherical distances between $\mathrm{X}$ and $\mathrm{Y}, \Delta \mathrm{S}_{2}$ is the spherical distance between $\mathrm{X}$ and $\mathrm{Z}$, while $\psi_{1}$ and $\psi_{2}$ are the azimuths of spherical paths $\mathrm{XY}$ and $\mathrm{XZ}$.

$\Phi=\arctan \left(\frac{\Delta \mathrm{t}_{1} \cdot \Delta \mathrm{S}_{2} \cdot \cos \psi_{2}-\Delta \mathrm{t}_{2} \cdot \Delta \mathrm{S}_{1} \cdot \cos \psi_{1}}{\Delta \mathrm{t}_{2} \cdot \Delta \mathrm{S}_{1} \cdot \sin \psi_{1}-\Delta \mathrm{t}_{1} \cdot \Delta \mathrm{S}_{2} \cdot \sin \psi_{2}}\right)$

Phase velocity of the TIDs was computed using

$$
\mathrm{V}=\frac{\Delta \mathrm{S}_{1}}{\Delta \mathrm{t}_{1}} \cos \left(\Phi-\psi_{1}\right)
$$

Different observation points of $\mathrm{X}, \mathrm{Y}$, and Z were chosen to compute absolute values of V and $\Phi$; thereafter we take the average value of $\mathrm{V}$ and $\Phi$ as the MSTIDs propagation velocity and azimuth. One important criterion that must be noted for computation of azimuth using equation (11), is that each of the GPS receiver stations within a sub-network must see the same satellite per observation time. Hence, the same satellite that could be seen by a sub-network is filtered for computation while other satellites are discarded. We also calculated the MSTIDs percentage occurrence rate (POR) of the event using equation (13).

$$
\mathrm{POR}=\left[\frac{\alpha}{\omega}\right] \times[100]
$$

where $\alpha$ is the total count number of dTEC estimation above ETH per epoch, $\omega$ is the total count number of dTEC estimation per epoch.

\subsection{Results}

We have analyzed the derived dTEC in the North African region during 2008-2016 and the wave-like structures depicted to be MSTIDs were estimated. The MSTIDs percentage occurrence rate (POR) was determined and divided into hourly bins. Variations in local time (LT) are analyzed by sorting the data into one-hour bins. Following Jayawardena et al. (2016) and Wang et al. (2017), we considered the daytime (DT: 0600-1800 LT) as dawn to dusk while the nighttime (NT: 1800-0600 LT) as dusk to dawn. However, for easy analysis and convenience, we converted the LT to universal time (UT) in a case where MSTIDs event are being observed simultaneously at more than one station in different sub-region. MSTIDs are observed and their characteristics are determined. Fig.4 shows an illustration for the case of a single day. 


\subsection{Observation of MSTIDs during 07 March 2010}

In this section we determine the MSTIDs characteristics for $7^{\text {th }}$ March 2010 (DOY 066) using equations (10), (11), and (12).
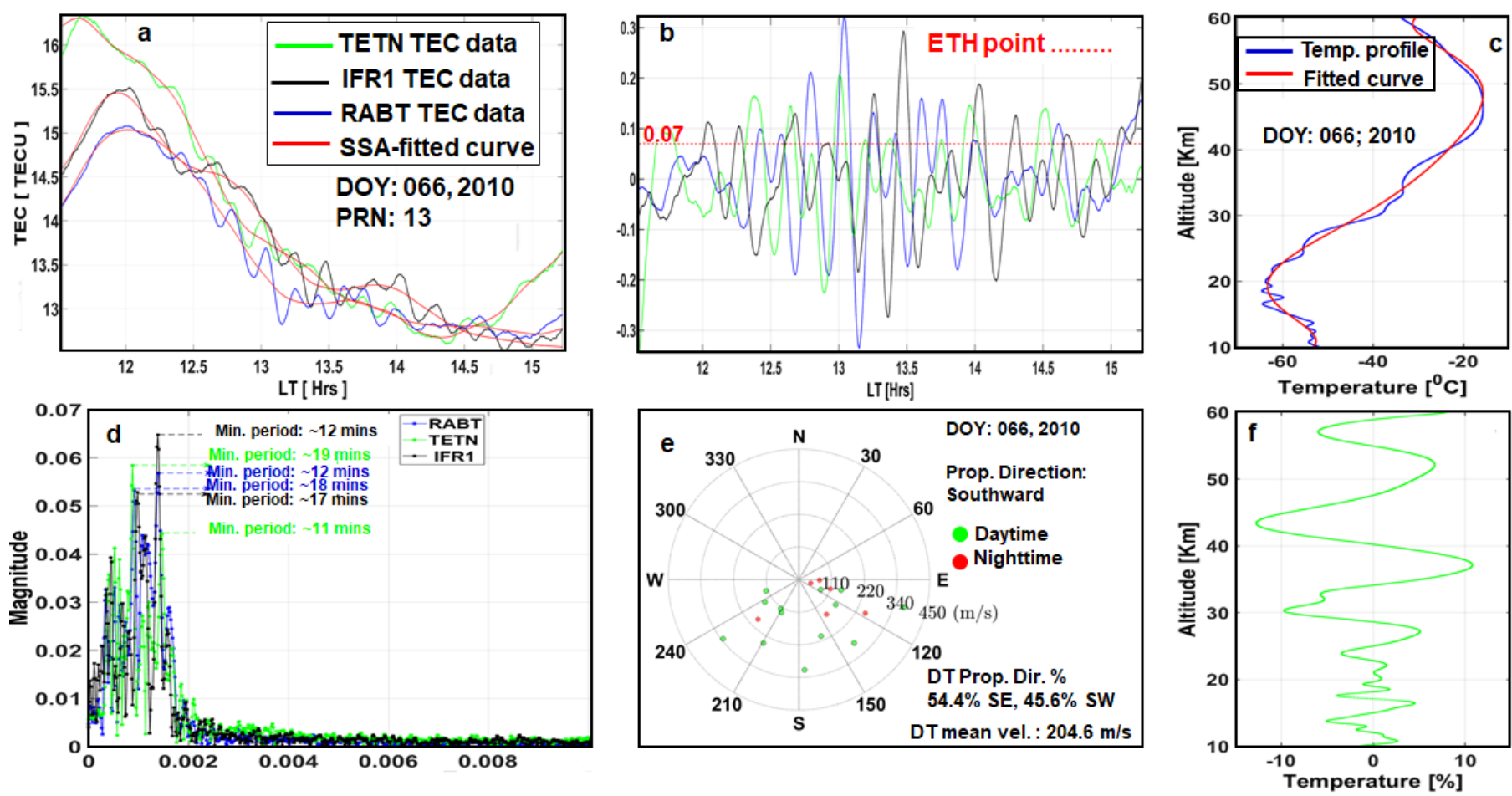

Fig. 4 (a) TEC versus UT measured by the GPS receivers (RABT, TETN and IFR1); color blue, green and black signal traces represent TEC values from the three receivers and the red lines represent the estimated background/unperturbed TEC values. (b) Corresponding detrended TEC time series of fig. (a), (c) Perturbed temperature profile from COSMIC satellite (blue color) and its fit (red color), (d) MSTIDs minimum and maximum dominant periods, (e) polar plot representing MSTIDs velocities and azimuth for daytime during DOY 066 (f) Signature of upward AGW propagation obtained from the detrended temperature profile in fig. (c).

The TEC time series exhibited continuous fluctuations as observed in PRN 13 at different local times (LT) in different stations located within the same sub-region. We used COSMIC temperature profile measurement within the geographic coordinate (lat: $31.4^{\circ} \mathrm{N}-32^{\circ} \mathrm{N}$, long: $1.7^{\circ} \mathrm{E}-1.8^{\mathrm{o}} \mathrm{E}$ ) that is most aligned or close in distance to the geographic area of interest. The DOY 066 daytime phenomenon in Fig. (4a) has majorly been thought to be caused by AGW as stated in the introductory section, and the perturbed temperature profile in Fig. (4c) characterizes a possible passage of AGWs from the troposphere to the ionosphere, and which eventually propagate above $50 \mathrm{~km}$ into the ionosphere (Azeem and Barlage, 2017). One of the important atmospheric parameters that exhibit the AGWs passage is the cloud top brightness temperature which ranges (i.e. threshold) between $-65^{\circ} \mathrm{C}$ and $-20^{\circ} \mathrm{C}$ during convection activities (Figueiredo et al., 2018). Having set the threshold for convection activities, we may then state that temperature lower than $-55^{\circ} \mathrm{C}$ on this day (DOY 066) could infer the convection activities, and hence a possible source of 
MSTIDs (Figueiredo et al., 2018). Following Jonah et al. (2016), the plotted Fig. (4c) indicates how the perturbed temperature profile exhibits the AGWs passage from the troposphere to the ionosphere. The wave on reaching the mesosphere (above $50 \mathrm{~km}$ ) breaks and release momentum where some waves travel further into the thermosphere (Tsuda et al., 2015), this brief process suggests a possible source of the MSTIDs during the selected day. Unfortunately, the COSMIC satellite could not capture temperature measurements above $60 \mathrm{~km}$ altitude, and this is one of the limitations of the COSMIC satellites for temperature measurement. The minimum and maximum dominant period of MSTIDs is obtained using FFT and it is computed to be an average of 11.7 mins and 18 mins, respectively. Fig.4 (e) shows that MSTIDs propagates towards the equator (southward) but indicated a higher percentage towards the south-east (SE). The temperature profile is detrended, in line with Wang et al. (2009), describing the output structure to be the vertical signature of upward AGW propagation. Fig.4(f) exhibited an increase in amplitude with height and it is a major characteristic of AGWs (Jonah et al., 2016).

\subsection{Two-dimensional observation of MSTIDs over North Africa}

Fig.5 shows the two-dimensional maps of MSTIDs over North Africa region at selected times during 1019 to 1200 UT (daytime) of day 066, 2010, using PRN 20 in all the eight stations. With careful observation, Fig.5 (a-b) shows an example of two-dimensional maps of TEC perturbations during the passage of MSTIDs over North African with maximum amplitude of 0.3 TECU. We splitted Fig.5 (a-b) into two to increase the map resolution.
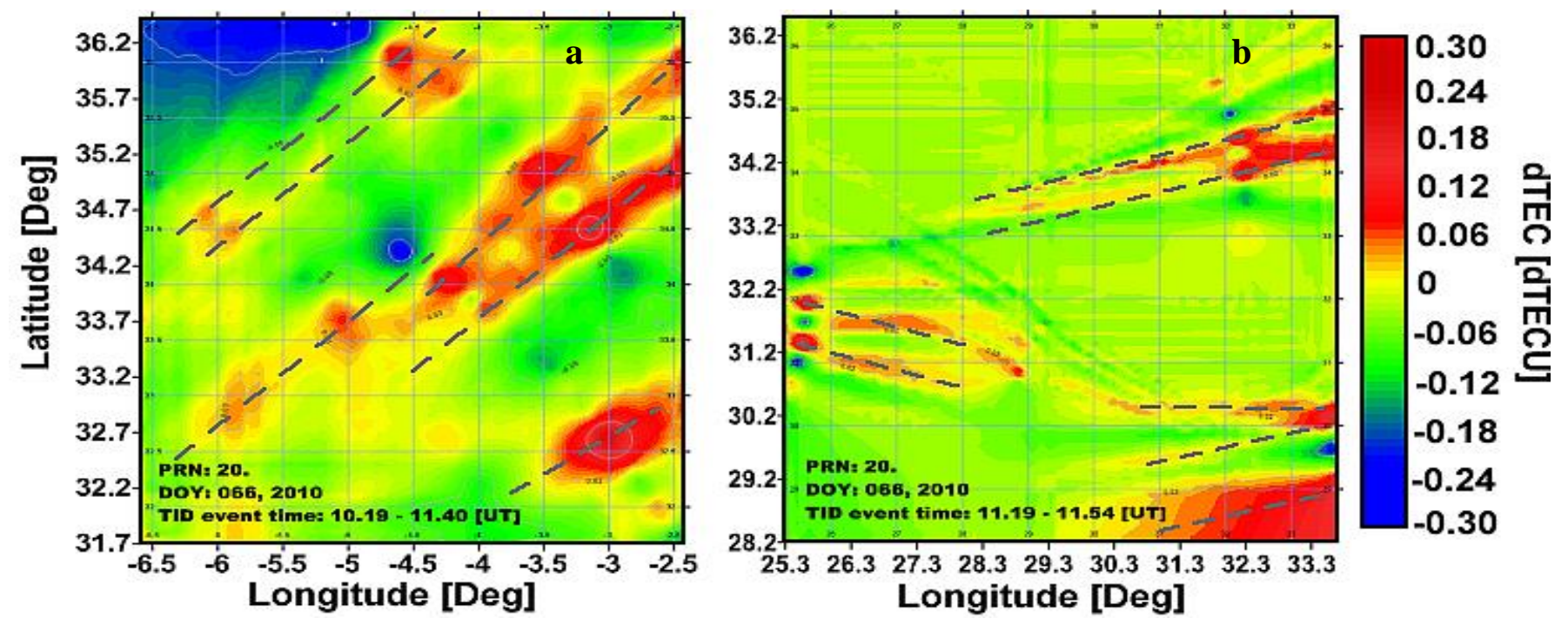

Fig. 5 Two-dimensional maps of MSTIDs over North Africa at 1019 to $~ 1200$ UT on $7^{\text {th }}$ March, 2010 (DOY 066).

The GPS receiver stations situated at the northwest have a close intra-distance than stations at the northeast, and this is the reason why Fig. (5a) produce a better MSTIDs formation and resolution than Fig. (5b). 


\subsection{Local observation of MSTIDs over selected GPS receiver stations in North Africa}

Figure 6(a-b) exhibits local diurnal and seasonal variations of MSTIDs occurrence at the different GPS receiver's stations located at mid-latitude stations. Data gap are indicated by the white portions on figure. Each station in each panel exhibited a similar contour structure but clearly shows different occurrence rate in terms of season and local time. In Fig. 6(a), the MSTIDs occurrence shows a strong dependence on the season (June solstice) and local times but with a major peak around the (nighttime) 2100-0200 LT ( 40 \% to 50 \%). Also, the daytime MSTIDs exhibited some minor peaks in December solstice around 1200-1600 LT. In Fig. 6(b), the nighttime MSTIDs occurrence exhibited similar seasonal (June solstice) and local times features as Fig. 6(a) but during 1900-0200 LT ( 30 \% to 40 \%). In addition, the daytime (09000-1600 LT) MSTIDs exhibited some peaks but not as pronounced as Fig. 6(a) during 2011 - 2015. Nighttime MSTIDs seems to decrease with an increasing solar activity. All stations exhibited an increasing MSTIDs POR consistently with the solar cycle. The highest MSTIDs POR at the Mid-latitude is consistently observed in June solstice during 2008-2016. The POR density shows that the occurrence rate varies with time of the day and season. This result seems to reveal occurrence variation and a level of inconsistency during day and night time from year to year. Hence, in subsequent section, we analyze day and nighttime amplitudes

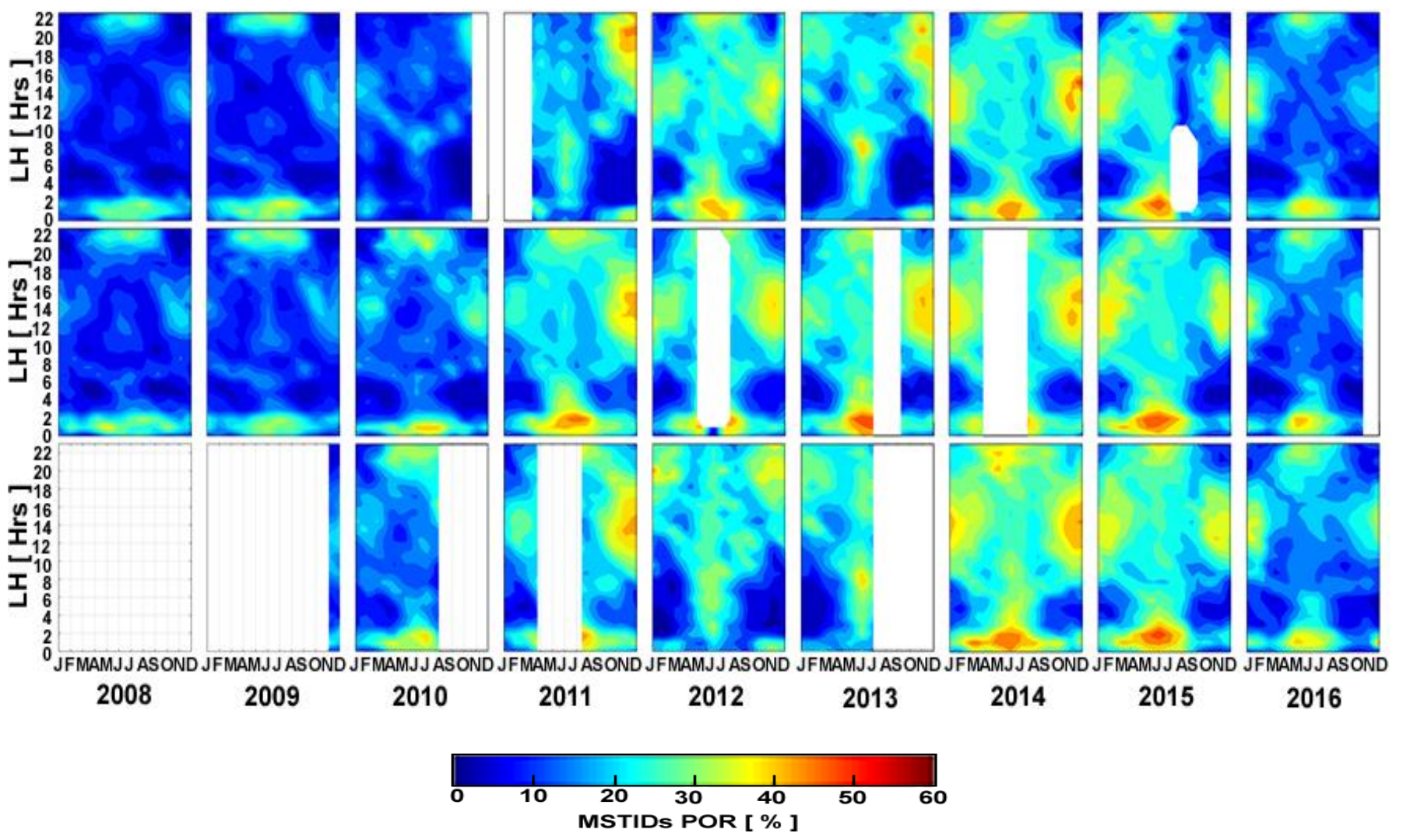

Fig. 6a: Local diurnal and seasonal variations of MSTIDs occurrence at different stations. White portions indicate data gap. Top panel is TETN station, Middle panel is RABT station, and bottom panel is IFR1 station 


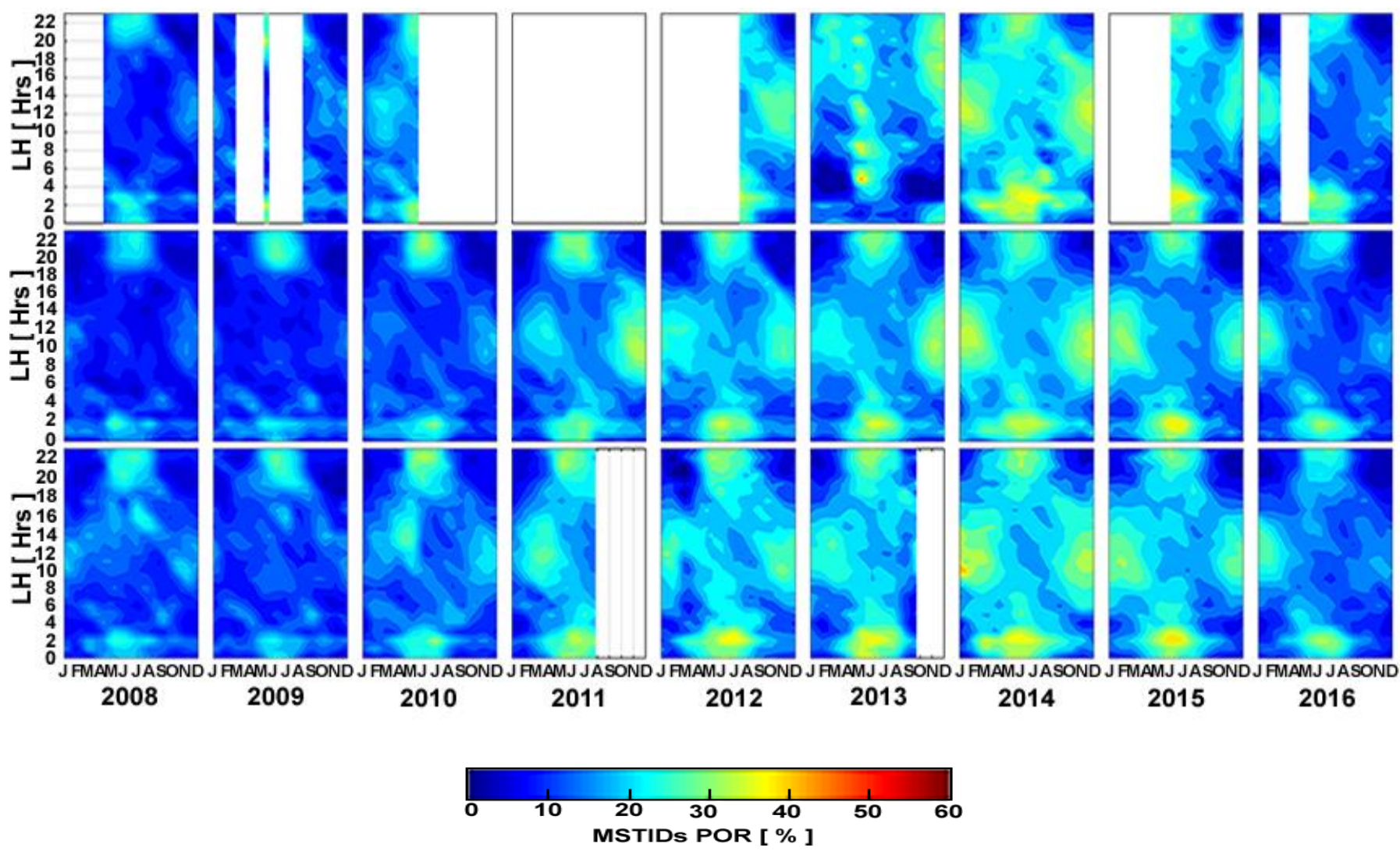

Fig. 6b: Local diurnal and seasonal variations of MSTIDs occurrence at different stations. White portions indicate data gap. Top panel is ALX2 station, Middle panel is NICO station, and bottom panel is RAMO station

\subsection{Interannual and seasonal dependence of MSTIDs amplitudes}

MSTIDs daily maximum amplitudes obtained from all stations were at mid-latitude were analyzed in this section. Figure (7) shows MSTIDs daily maximum amplitudes for daytime and nighttime. For better visual analysis and to observe slightest changes in the multiple scatter plots, we introduced a mathematical function (simple moving average) which estimates the average value to determine the trend line-curve for both day and night (red and blue line) which we use for analysis. Both nighttime and daytime exhibited similar pattern of trend curve but different amplitude variability. For instance, the nighttime amplitude consistently and dominantly higher than daytime during the solar minimum year (2008-2010), having a high peak around (0.22 - 0.37 TECU) in June solstice. 


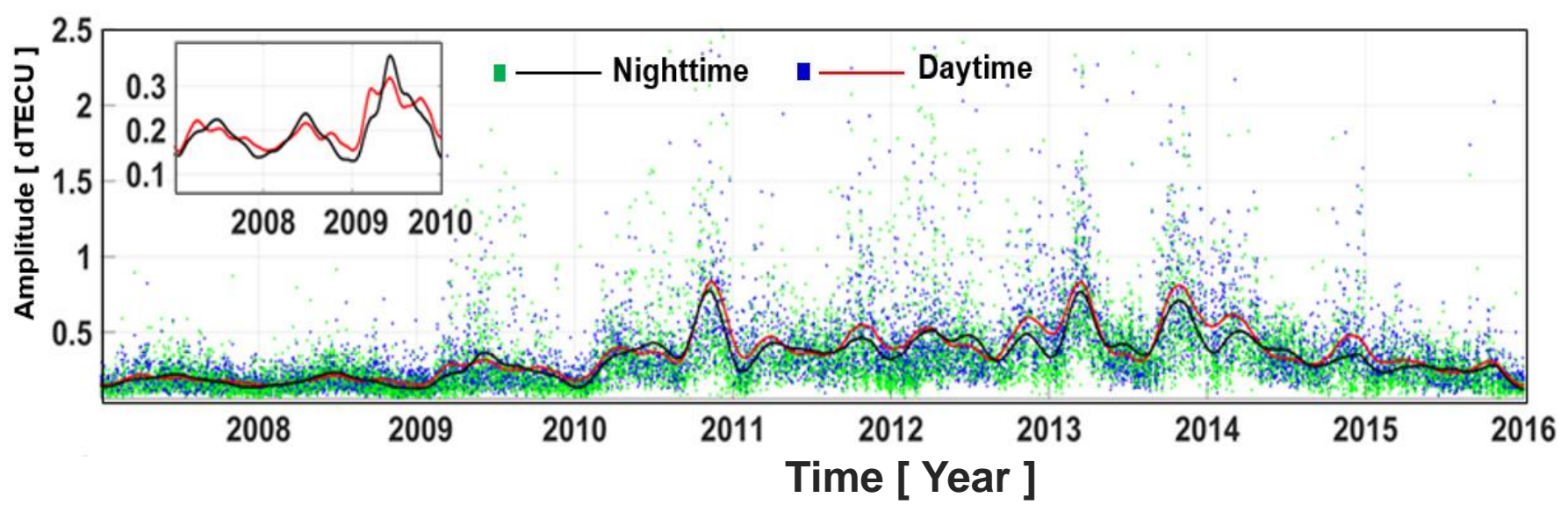

Fig. 7: MSTIDs amplitude time series for both nighttime and daytime

The high peak amplitudes switched from nighttime to daytime, exhibiting major peaks around (0.45 - 0.94 TECU) in September equinox during 2011 - 2015, and March equinox of 2014. The nighttime amplitude consistently exhibits higher peak during the June solstice, while the daytime consistently exhibits higher peak during the equinox months during 2008-2016. The dominant major higher peaks are observed in solar maximum year of 2014. By considering the solar minimum and maximum years, the nighttime amplitude seems to be slightly decreasing with increase in solar activity during June solstice. The daytime amplitude values increase with solar activity. However, it must be noted that the high background TEC experienced during high solar activities in equinox season also could influence the high MSTIDs amplitude, in that whenever the TEC background is large, the amplitude of TEC perturbation is also large.

\subsection{MSTIDs characteristics}

In estimating the MSTIDs azimuth, we followed equation (11) in section 3.3, and we choose to focus only on stations with the closest intra-distance between one another. Hence, we selected GPS receiver stations (RABT-TETN-IFR1) which have an estimated intra-distance of $206 \mathrm{~km}$, while other stations have their intra-distance more than $600 \mathrm{~km}$. Fig. 8 (top panel) shows polar plots representing MSTID velocities (in m/s) and azimuths during 2008 - 2016 for March equinox, June solstice, September equinox, and December solstice. In other to estimate a discrete propagation direction as a function of percentage since the polar measurements looks clustered, and for clearer analysis, we further divided the azimuth measurements into daytime (DT) and nighttime (NT) and get it plotted on a bar-chart (Fig. 8 (bottom panel)). The bar-chart shows discrete cardinal directions; North (N), North-East (NE), East (E), South-East (SE), South (S), SouthWest (SW), West (W), and North-West (NW) following Otsuka et al. (2013) approach, the bar chart also shows the daytime and nighttime mean velocity for each of the seasons. The MSTIDs propagation velocity 
is within $50-450 \mathrm{~m} / \mathrm{s}$, with velocity dominance of $200-300 \mathrm{~m} / \mathrm{s}$ for every season except September equinox which has a dominance velocity value between $100-200 \mathrm{~m} / \mathrm{s}$.
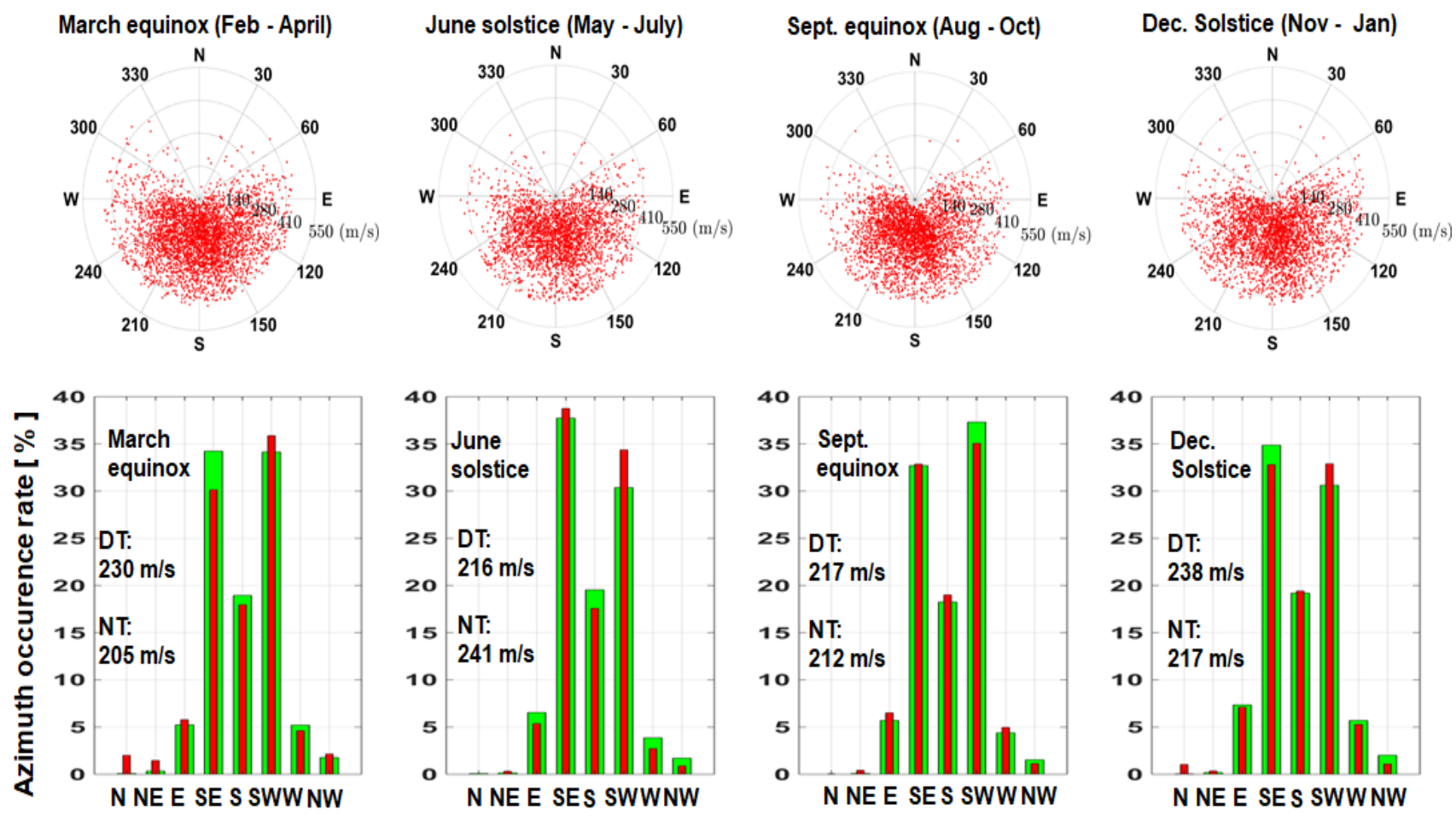

Azimuth

Nighttime (NT) Daytime (DT)

Fig. 8: (top panel) shows polar plots representing MSTID velocities (in $\mathrm{m} / \mathrm{s}$ ) and azimuths for different seasons. (bottom panel) Bar chart showing cardinal directions of MSTIDs propagation having the percentage azimuth occurrence rate on the vertical axis, while the corresponding cardinal directions are on the horizontal axis.

Generally, the entire MSTIDs dominantly propagates southward (equatorward) as seen in Fig. 8 (top panel), dominantly between $120^{\circ}-230^{\circ}$. However, there are slight variations in propagation direction during daytime and nighttime as seen in Fig. 8 (bottom panel) which reveals the preferred propagation direction. Some few MSTIDs is observed to propagate northward, but most observation are seen to be dominantly southeastward and southwestward for both daytime and nighttime MSTIDs in all the seasons but with slight exceptional cases in March equinox, June solstice and December solstice, where the nighttime MSTIDs propagation towards the southwest is slightly higher than the daytime by $0.7 \%, 1.3 \%$ and $4.0 \%$, respectively. In addition, the daytime MSTIDs propagates towards the southeast, and slightly higher than the nighttime which also propagates in the same direction by $\sim 4.0 \%$ during the March equinox and December solstice, respectively. The nighttime MSTIDs percentage of propagation direction is higher in both southeast and southwest direction during June solstice, and during the September equinox the daytime MSTIDs percentage of propagation direction is higher southwest direction while the nighttime is slightly higher than the daytime in southeast direction. The detrended TEC time series were used to obtain the MSTIDs period by using fast Fourier transform (FFT) following (Husin et al., 2011; Arikan et al., 2017). 
The MSTIDs occurrence periods estimated with less than 6 minutes were regarded as noise fluctuations and therefore eliminated (Valladares and Hei, (2012)). The velocities were computed using equation (12) and the wavelengths were estimated from the distance the TEC wave-like structure traveled in space (latitude or longitude) following (Jonah et al., 2016). The daytime MSTIDs mean velocity is larger than the nighttime in all season, except in June solstice where the MSTIDs velocity experiences a reverse case. All seasons exhibited similar mean MSTIDs occurrence period (DT: 14 - 38 mins, NT: 13 - 35 mins) and wavelength (DT: 118 - $391 \mathrm{~km}, 96$ - $382 \mathrm{~km}$ ). The estimated values for velocity, period, and wavelength, respectively are within the ranges typically associated with MSTIDs discussed in section (1.0). The regional distribution of MSTIDs on a spatio-temporal map over the mid-latitude of North Africa region is shown in Fig. (9). MSTIDs maps from different sectors at mid-latitude were superimposed. The local time (LT) was converted to UT for time uniformity, easy analysis and most importantly to observe the dominant event time of occurrence for each year covering geographic latitudes (GL) $30^{\circ} \mathrm{N}$ to $42^{\circ} \mathrm{N}$ and longitude $18^{\circ} \mathrm{W}$ to $42^{\circ} \mathrm{E}$ (Otsuka et al., 2013).

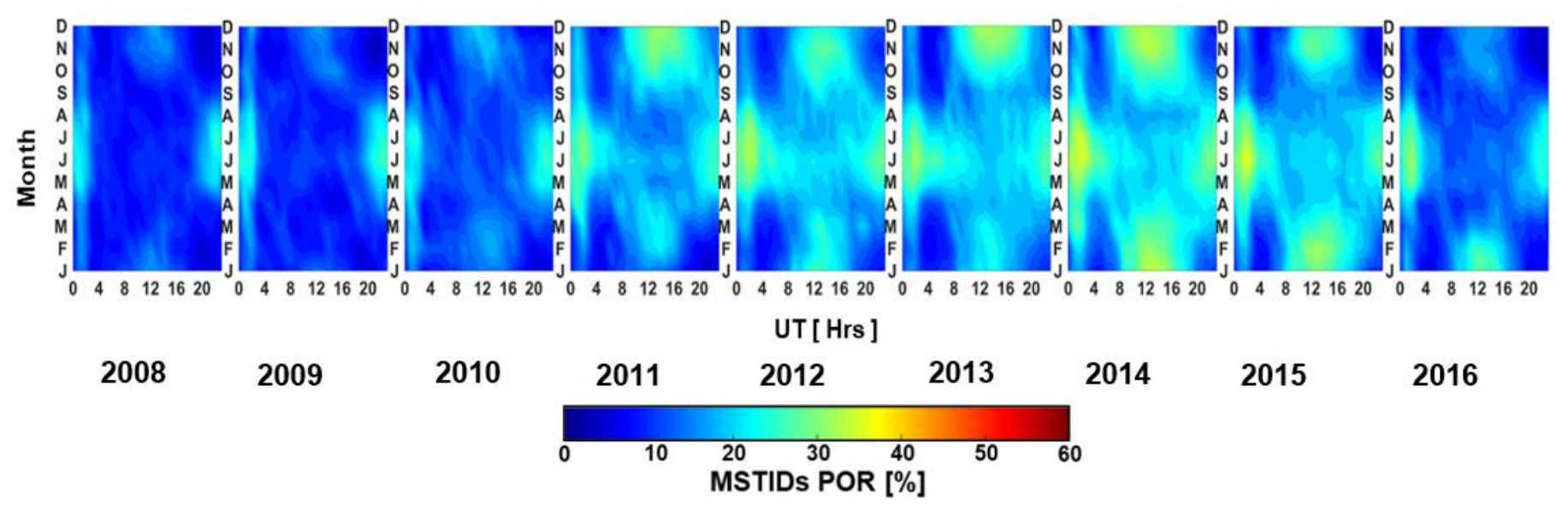

Fig. 9 Universal time and seasonal variations in MSTIDs POR at mid-latitudes $\left(42^{\circ} \mathrm{N} \leq \mathrm{GL} \geq 30^{0} \mathrm{~N}\right) ; 2008-2016$

The distribution of dominance occurrence of MSTIDs in Fig. (9) shows a semiannual variation with the major primary peak at June solstice (i.e. summer) during the NT (2100 - 0300 UT) and secondary peak at December solstice (i.e. winter) during the DT (1000 - 1500 UT). The maximum MSTIDs POR was observed to be $\sim 45 \%$ in 2014 and 2015.

\subsection{Discussion}

We have investigated statistically dTEC variations observed by GPS receivers located in Northern African region at mid-latitudes to reveal MSTIDs occurrence rate at local time, seasonal, latitudinal variations and propagation direction at daytime and nighttime, respectively, during 2008-2016. 
Our statistical results show a distinct difference between the observed MSTIDs activities. Fig. (3a) and (4a) shows daytime TEC measurement exhibiting wave-like structures depicting to be MSTIDs due to the passage of AGW (Hines, 1960; Hooke, 1968; Jonah et al., 2016; Oinats et al., 2016; Valladares et al., 2012). The AGWs passage involves vertical displacement of air parcels originating in the troposphere (Hines (1960)) and which causes perturbation in the ionospheric electron density. Temperature and wind perturbations are the two parameters that oscillate for a freely propagating wave which transport energy and momentum from their source into certain height in the ionosphere. The neutral air wind perturbation collides with the plasma at $\mathrm{F}$ region, and then the charged ions are set in motion but are constrained to move along the magnetic field lines. The transportation of the charged molecules/ions along the magnetic field lines leads to electron density enhancement in certain places along the wave-front and also depletions in some other places. The continuous and regular enhancement and depletion of the plasma density consequently leads to TIDs occurrence (Hooke, 1968).

The observation in Fig. (4a) shows a TEC wave-like structures (disturbed TEC) which could possibly be as a consequence of AGWs passage in investigated day, Fig. (4c) shows the disturbed temperature profile obtained from COSMIC satellite and Fig. (4f) shows the signature of upward AGW propagation as obtained from the detrended temperature profile. Our result in Fig 4(a-f) is similar with the results obtained by Jonah et al. (2016) who reported MSTIDs occurrence and causative mechanism in the Southern hemisphere of the Brazilian sector. The MSTIDs 2-D map in Fig. 5 (a-b) shows TEC perturbations during the passage of MSTIDs, which seems to stretch from the Northwest (NW) towards the Northeast (NE) with a maximum amplitude peak value of 0.30 dTECU, this elongation from NW to NE confirms the MSTIDs feature of long-distance travel or propagation hypothesis of MSTIDs (Frissell et al., 2014).

Fig. 6 (a) and Fig. (b) consist of GPS receiver stations located at NW and NE, respectively, and they show different characteristics between daytime and nighttime MSTIDs occurrence, such as local time, seasonal, and solar activity dependence. These facts indicate that different mechanisms initiate MSTIDs occurrence during daytime and nighttime period, and at different seasons. A high occurrence rate of MSTIDs was observed in the daytime during 1100-1600 LT, and 0900-1400 LT at NW and NE, respectively during the March equinox and December solstice, respectively. However, the nighttime MSTIDs exhibited highest occurrence rate observed in June solstice during 2100 - 0200 LT and 1900 - 0200 LT at NW and NE, respectively. Both daytime and nighttime MSTIDs occurrence at NW is more pronounced during 20112015, than SE stations. The daytime MSTIDs POR increases with increase with solar activity. The nighttime during 0001- 0200 LT seems to increase with increase in solar activity, while the nighttime during 1900 2300 LT seems to decrease with increases in solar activity. Our MSTIDs seasonal occurrence results show a good agreement with the MSTIDs investigation conducted by Tsugawa et al. (2006a) who reported MSTIDs 
occurrence over South-East Asian sector (Japan). In their investigation, they reported nighttime (2100 0300 LT) MSTIDs to be the highest activities in every year during summer (May-August), they added that the summer nighttime activities become weaker as the solar cycle approaches its maximum. They also observed on the contrary that the daytime (0900 - 1500 LT) MSTIDs occurrence is high during the winter, but there is no clear indication of solar activity dependence of the daytime activities. The slight difference between this current study and Tsugawa et al. (2006a) is that the current study shows a clear increase in daytime MSTIDs occurrence as solar activity increases, and the nighttime MSTIDs seems to be decreasing specifically during 1900 - 2300 LT as solar activity increases. Furthermore, the seasonal results in this current study is similar to the result obtained from with MSTIDs study over the North American sector (California) conducted by Hernández-Pajares et al. (2012), they reported daytime MSTIDs occurrence during winter (November-January) and fall (August-October), and nighttime during summer (May-July) and spring (February-April), whereas this current study report daytime and nighttime occurrence during December solstice and June solstice, respectively. However, the nighttime (0001 - 0200 LT) slightly extends to March equinox season during the solar maximum of 2014.

In Fig. (7), each year of the MSTIDs amplitude time series exhibited an asymmetric structure, and most especially during the daytime period. The MSTIDs occurrence exhibited a significant increase in the year 2011 relative to 2009 - 2010, and 2012 - 2013, during September equinox, possibly due to the increase in solar activity as expressed by an increase in sunspot numbers. The mean sunspot numbers in September equinox in 2009, 2010, 2011, 2012, and 2013 are 4.9, 33.2, 104, 88, and 87, respectively (see. http://www.sidc.be/sunspot-data/) (Tariku (2015)). Generally, the MSTIDs amplitude increase with an increase in solar activity, most especially the daytime period, this result agrees with Oinats et al. (2016) who investigated MSTIDs observation over Hokkaido East during 2007 - 2014, and over European-Asian sector during the 2013-2014 using radar data. They reported an increase in amplitude with an increase in solar activity, and that the amplitude tends to increase with increasing auroral electrojet (AE) index, and also found that MSTIDs amplitude is dominantly high at daytime.

In estimating the MSTIDs propagation direction, we grouped the azimuth of the MSTIDs propagation direction into daytime, nighttime and seasons. The propagation direction of MSTIDs for both daytime (DT) and nighttime (NT) are dominantly Southward (equatorward) as observed from the Northern hemisphere in Fig (4e), and Fig. (8-top panel), while Fig. (8-bottom panel) show the azimuth occurrence rate expressed in percentage following Otsuka et al. (2013) approach. Some previous studies have shown agreement or similar result with the current result of propagation direction of MSTIDs. For instance, Jacobson et al. (1995) investigated daytime and nighttime MSTIDs occurrence using a very long baseline interferometer 
(VLBI) array illuminated by $136 / 137-\mathrm{MHz}$ radio beacons of two geostationary satellites at New Mexico $\left(35.9^{\circ} \mathrm{N}, 106.3^{\circ} \mathrm{W}\right)$, and they reported different seasonal variations in terms of occurrence rate and their propagation direction. They further showed that the preferred daytime MSTIDs propagation direction is southward during winter and equinox seasons, respectively, while the nighttime MSTIDs often occur during summer solstice during, autumn equinox and propagate toward the west/northwest. In addition, Kotake et al. (2007) reported the azimuth of the MSTID propagation direction during the daytime to be southeastward $\left(90^{\circ}\right.$ to $\left.240^{\circ}\right)$ in equinox and in winter $\left(120^{\circ}\right.$ to $\left.240^{\circ}\right)$ season, respectively, and also reported the nighttime MSTIDs to be southwestward and westward propagation (between $210^{\circ}$ and $300^{\circ}$ in azimuth) in equinox and summer seasons. While Ding et al. (2011) reported a dominant propagation of daytime MSTIDs towards the equator, adding that nighttime and daytime MSTIDs are similar in period and propagation azimuth. However, they stated that the nighttime MSTIDs dominantly propagated southwestward in the Northern hemisphere at all seasons, irrespective of the latitude, and also the nighttime MSTIDs highest propagation occurrence in June, around the summer solstice. In this current study, both the daytime and nighttime MSTIDs are dominantly propagating southward (rotating clockwise from $90^{\circ}$ to $240^{\circ}$ ), but spread into different cardinal directions. Figure (8-bottom panel) exhibited a discrete propagation direction for daytime and nighttime, and it shows the dominant daytime MSTIDs exceeded the nighttime by $\sim 4$ in the southeastward propagation during March equinox and December solstice. This result shows similarity with the seasonal propagation dominance obtained by both Jacobson et al. (1995) and Kotake et al. (2007) discussed earlier, but differs in terms of propagation direction with Jacobson et al. (1995). The southwestward dominant propagation direction occurrence for nighttime MSTIDs during March equinox, June solstice, and December solstice which is also similar to Kotake et al. (2007), and Ding et al. (2011), except for December solstice. Furthermore, this study shows June solstice to have the highest percentage of azimuth of the nighttime MSTID propagation direction which agrees with Ding et al. (2011), but the preferred propagation direction is southeastward. Figure (8- bottom panel) also shows MSTIDs preferably propagating towards the $\mathrm{N}, \mathrm{NE}, \mathrm{E}, \mathrm{W}$, and $\mathrm{NW}$, but the percentage of azimuth of the propagation direction is below $6 \%$ which could be as a result of local background geophysical conditions during the propagation period. Our result shows that not all daytime and nighttime MSTIDs propagates southeast and southwest, respectively, as it has been hypothesized by some previous studies, and based on the comparison of the MSTIDs propagation direction in the current study with previous propagation direction reports by different authors we may then infer that mechanisms causing the azimuth of the MSTID propagation direction could depend on the location, local time, season, background geophysical conditions and prevailing ionospheric conditions over the geographical location of interest.

The mean propagation velocity of MSTIDs varies from $205-241 \mathrm{~m} / \mathrm{s}$, with the daytime propagation velocity mostly higher than nighttime, which is similar with previous study (Husin et al., 2011; Hernandez- 
Pajares et al., 2012) except during June solstice where the nighttime is higher than the daytime which agrees with Oinats et al. (2016). The major contrast between present study and Oinats et al. (2016) is that, the nighttime MSTIDs propagation velocity is higher than the daytime only in June solstice, on the contrary Oinats et al. (2016) reported higher daytime velocity values over in the nighttime during 2007-2014, using HF radar data.

However, several studies have been done to investigate the mechanisms responsible for the daytime and nighttime propagation direction of MSTIDs. Heisler, (1963) stated that propagation direction of TIDs is towards the ionospheric part where it is mostly illuminated by the Sun (from the Northern hemisphere), whereas Thome, (1964) stated that the most supported theory for propagation direction is that TIDs propagates in the direction of the geomagnetic field lines. Hooke, $(1968,1970)$ in his investigation on ionospheric response to internal gravity waves stated that at F-region heights, the ions move and travel along the geomagnetic field lines through neutral-ion collision, with a velocity the same as the velocity of the neutral motion along the geomagnetic field caused by the gravity waves, during this process some azimuthal directions of wave propagation are preferred as a function of the ionospheric response that are evoked. However, the motion of the ions across the magnetic field line hypothesized by Thome, (1964) is constrained to move along the magnetic field lines because the gyro-frequency of the ions is much higher than the frequency of the ion-neutral collisions. The direction of the motion of the ions consequentially leads to directivity in the response of the electron density variations to the gravity waves. This kind of directivity phenomena could be responsible for daytime MSTIDs southward propagation direction (Kotake et al., 2007). Besides, an anisotropic frictional ion drag force has been thought to also responsible for the Southward propagation of the daytime MSTID direction (Liu and Yeh, 1969; Kelley and Miller, 1997).

The nighttime MSTID were previously found to be associated with increases in the F-region peak electron density altitude by Behnke (1979), and its source was conventionally assumed to be generated by electrodynamical forces such as Perkins instability (Perkins, 1973; Kelley and Fukao, 1991; Kelley and Miller, 1997; Garcia et al., 2000; Tsugawa et al., 2007; Otsuka et al., 2007). Perkins created a mid-latitude ionospheric model that finds a way around solving the complexity of the mid-latitude ionosphere. In his model he solved the momentum equations by integrating quantities along the magnetic field line and under certain conditions found the existence of unstable modes when a North-South electric field or Eastern neutral wind existed, and consequently, a pre-existent irregularity will grow with wave-fronts oriented from North-West to South-East in the Northern Hemisphere (Perkins, 1973). These instabilities are often strong in the nighttime and can generate local polarization electric field that can move the plasma upward (downward) via the E x B drift, which consequently causes perturbation in the plasma density. It must be noted that the growth rate of the generative mechanism of Perkins instability at mid-latitudes is very low 
and therefore would require additional seeds such as gravity waves (Hunsucker, 1982; Huang et al., 1994) as well as electrodynamic coupling processes between F- and E- regions to boost the low Perkins growth rate to allow for the MSTIDs development (Cosgrove, 2004; Otsuka et al., 2007; Yokoyama and Hysell, 2010). The Perkins instability theory is responsible for the peak of nighttime MSTIDs at the June solstice which is inversely proportional to the neutral density. It also predicts the MSTIDs alignment with respect to the background magnetic field, but it incorrectly predicts the direction of phase front (Perkins, 1973).

The MSTIDs distribution map is developed in Fig. (9), the map gives a general view of MSTIDs in the North Africa region, and most importantly the MSTIDs occurrence dominance. The regional distribution has similar features such as season and time (Hrs) of occurrence as Fig. (6a-b) but with little difference in occurrence time range, and solar activity dependence. The high MSTIDs occurrence level observed during daytime and nighttime are 0900-1600 UT (December solstice (winter)) and 2000-0400 UT (June solstice (summer) respectively, in each year but the seasonal peak got extended to March equinox during in 2011, 2014, and 2015, but more pronounced in 2014. The same occurrence mechanism discussed above for local sector is also responsible for the regional distribution. The figure shows a consistent increase in MSTIDs occurrence with increase solar activity.

It must be noted that the disturbed temperature profile measurement which characterizes the passage of AGWs used in this study is limited in altitude, in that the temperature profile data from COSMIC satellite do not exceed $60 \mathrm{~km}$ in altitude. This known fact is one of the limitations of the COSMIC satellites' temperature profile. Therefore, temperature profile data from sounding of the Atmosphere using Broadband Emission Radiometry (SABER) could be used as an alternative for the COSMIC-RO temperature profile deficiency since SABER satellite could produce temperature profiles measurements with altitude up to 100 $\mathrm{km}$. This investigation can be carried out in future work.

\section{Conclusions}

For the first time, the climatology of MSTIDs has been studied during solar cycle \#24 (2008-2016) using the GPS network within the North African sector at the Northern Hemisphere. Quiet days with Kp $\leq 3$ were considered in this study. We examined the MSTIDs occurrence rate, statistical characteristics for daytime and nighttime, the excitation mechanism and spatial-temporal distribution. The study concluded that: 
1. From the estimated TEC data, we derive the TEC perturbations (dTEC) by subtracting the TEC time series from corresponding best fitted (TECSSA-fit) obtained from non-parametric models such as SSA as a bandpass technique to filter out TEC perturbations associated with MSTIDs.

2. We deduced from the analysis of TEC wave-like structures and perturbed temperature profile of the selected day ( $7^{\text {th }}$ March 2010), that AGWs may be responsible for the MSTIDs occurrence. Also, MSTIDs exhibited the ability to propagate over long distances. Hence, MSTIDs have been observed by both short and long baseline measurements as in the case of NW and NE in Fig. 5(a-b) for the selected day.

3. MSTIDs occurrence is a local phenomenon and its occurrence rate is majorly dependent on local time, season and latitude. However, we found that MSTIDs occurrence rate during study period can categorized into different groups based on location. There is diurnal variability in MSTIDs occurrence at Mid-latitude, but the daytime MSTIDs dominantly occur around (NW: 1200-1600 LT) and (NE: 1000-1400 LT) in December solstice, while nighttime dominantly occurs around (NW: 2100-0200 LT) and (NE: 1900-0200 LT) in June solstice, but get extended to March equinox during solar maximum (2014).

4. MSTIDs occurrence rate were observed to increase during daytime and seems to decrease during nighttime with increase in solar activity.

5.Not all daytime and nighttime MSTIDs propagates southeast and southwest respectively at every season as reported by some previous studies.

6. The results show that both daytime and nighttime MSTIDs over North African dominantly propagate southward (equator). However, there are indications of dominant propagation ( 4\% more than nighttime) towards the southeast during daytime in March equinox and December solstice only, while in the nighttime the MSTIDs dominantly propagate towards the southwest during March equinox, June solstice and December solstice only. Some MSTIDs propagate towards other cardinal directions but in low quantity (below $6 \%$ ).

7.The mean phase velocity in daytime MSTIDs is higher than the nighttime in every season, except during June solstice.

8. There is a consistent asymmetrical effect of MSTIDs amplitude at both nighttime and daytime in equinox months. There is dominant annual asymmetry effect of MSTIDs in June solstice during solar minimum 
with nighttime amplitude higher than daytime, but the amplitude peak switched from June (nighttime) solstice to equinox seasons (daytime). The regional distribution of the MSTIDs activity shows a primary peak during June solstice and secondary peak during December solstice.

\section{Abbreviations}

MSTIDs: medium-scale traveling ionospheric disturbances; GPS: Global Positioning System; AGW: Atmospheric Gravity Waves; TEC: Total Electron Content; dTEC: TEC perturbations; NNSS: Navy Navigation Satellite System; SuperDARN: Super Dual Auroral Radar Network; HF: High frequency; COSMIC: Constellation Observing System for Meteorology, Ionosphere, and Climate; RO: radio occultation; OR: occurrence rate; LEO: low Earth orbit; CDAAC: COSMIC Data Analysis and Archive Center; DCB: differential code biases; VTEC: vertical TEC; SSA: singular spectrum analysis; FFT: fast Fourier transform; POR: percentage occurrence rate; LT: local times; UT: universal time; DT: daytime; NT: nighttime; AMEC: annual MSTIDs event count

\section{Availability of data and materials}

The datasets generated and/or analysed in support of the findings of this study are available upon request from the corresponding author.

\section{Competing interests}

The authors declare that they have no competing interests.

\section{Funding}

This work was financially supported by the Advance Technologies for Navigation and Geodesy (ADVANTAGE) project (grant number: ZT-0007) funded by Helmholtz-Gemeinschaft, Germany.

\section{Authors' contributions}

Oluwadare T Seun performed data processing, MSTIDs estimation, MSTIDs statistical analysis, discussed the MSTIDs mechanisms and drafted the manuscript. Norbert Jakowski and Cesar E. Valladares elaborated on MSTIDs mechanism and propagation direction respectively. Andrew O. Akala, Oladipo E. Abe, Mahdi M. Alizadeh, Harald Schuh participated in the interpretation of the MSTIDs results, proper use of technical language and sequential arrangement of manuscript text structure. All authors have contributed to the work of Oluwadare T Seun.

\section{Acknowledgments}

The authors thank the International GNSS Service (IGS), University NAVSTAR Consortium (UNAVCO), The Centre d'Etudes Alexandrines, Egypt, The African Geodetic Reference Frame (AFREF), and Constellation Observing System for Meteorology, Ionosphere, and Climate (COSMIC) for preserving the raw data and make it available for scientific uses. We also acknowledge the financial support from ADVANTAGE project (grant number: ZT-0007) funded by Helmholtz-Gemeinschaft, Germany. 


\section{Authors' information}

Oluwadare Temitope Seun.

German Research Centre for Geosciences GFZ, Telegrafenberg, D-14473 Potsdam, Germany. oluwa@gfz-potsdam.de, temitopeseun@yahoo.co.uk

\section{References}

Abe O E, Otero Villamide X, Paparini C, Radicella S M, Nava B, Rodríguez-Bouza M (2017), Performance evaluation of GNSS-TEC estimation techniques at the grid point in middle and low latitudes during different geomagnetic conditions, J Geod (2017) 91:409-417 DOI 10.1007/s00190-016-0972-z

Afraimovich E L, Palamartchouk K S, Perevalova N P (1998) GPS radio interfereometry of travelling ionospheric disturbances. J. Atmos. Sol. Terr. Phys., 60, 1205- 1223

Afraimovich E L, Boitman O N, Zhovty E I, Kalikhman A D, Pirog T.G (1999) Dynamics and anisotropy of travelling ionospheric distances as deduced from transionspheric sounding data. Radio Sci., 34, 477-487

Arikan F, Yarici A (2017) Spectral investigation of traveling ionospheric disturbances: IONOLAB-FFT. Geodesy and Geodynamics. 8 (2017), 297-304. http://dx.doi.org/10.1016/j.geog.2017.05.002.

Azeem I, Barlage M (2017) Atmosphere-ionosphere coupling from convectively generated gravity waves, Advances in Space Research. Pages 1931-1941, doi.org/10.1016/j.asr.2017.09.029

Bagiya M S, Joshi H P, Iyer K N, Aggarwal M, Ravindran S, Pathan B M (2009) TEC variations during low solar activity period (2005-2007) near the Equatorial Ionospheric Anomaly Crest region in India. Ann. Geophys. 27, 10471057

Behnke R (1979) F layer height bands in the nocturnal ionosphere over Arecibo. J. Geophys. Res., 84, 974-978, doi:10.1029/JA084iA03p00974

Bolaji O S, Adeniyi, J O, Radicella S M, Doherty P H (2012) Variability of total electron content over an equatorial West African station during low solar activity. Radio Sci. (USA) 47. doi.org/ 10.1029/2011RS004812, 2012

Chandra K R, Srinivas V S, Sarma A D (2009) Investigation of ionospheric gradients for GAGAN application. Earth Planet and Space

Chen Guanyi, Chen Zhou, Yi Liu, Jiaqi Zhao, Qiong Tang, Xiang Wang, Zhengyu Zhao (2019) A statistical analysis of medium-scale traveling ionospheric disturbances during 2014-2017 using the Hong Kong CORS network. doi.org/10.1186/s40623-019-1031-9, Earth, Planets and Space

Ciraolo L, Azpilicueta F, Brunini C, Meza C, Radicella S M (2007) Calibration errors on experimental slant total electron content (TEC) determined with GPS. J Geodesy

Ciraolo L, Azpilicueta F, Brunini C, Meza A, Radicella S M (2006) Calibration errors on experimental slant total electron content (TEC) determined with GPS, J Geodesy (2007) 81:111-120, DOI 10.1007/s00190-006-0093-1

Cosgrove R B (2004) Coupling of the Perkins instability and the sporadic E layer instability derived from physical arguments. J. Geophys. Res., 109. doi:10.1029/2003JA010295

Ding F, Wan W, Ning B, Wang M (2007) Large-scale traveling ionospheric disturbances observed by GPS total electron content during the magnetic storm of 29-30 October 2003. J Geophys Res, doi:10.1029/2006ja012013, 2007 
Ding F, Yuan H, Wan W, Reid I M, Woithe J M (2004) Occurrence characteristics of medium-scale gravity waves observed in $\mathrm{OH}$ and $\mathrm{OI}$ nightglow over Adelaide (34.5 $\left.{ }^{\circ} \mathrm{S}, 138.5^{\circ} \mathrm{E}\right)$. J Geophys Res. doi.org/10.1029/2003JD004096

Ding F, Weixing W, Guirong X, Tao Y, Guanlin Y, and Jing-Song W (2011) Climatogy of medium-scale traveling ionospheric disturbances observed by a GPS network in central China, J Geophys Res. doi: 10.1029/2011JA016545

Fedorenko Y P, Tyrnov O F, Fedorenko V N (2010) Parameters of Traveling Ionospheric Disturbances Estimated from Satellite Beacon Observations in Low Earth Orbit. doi.org/10.1134/S0016793210040109

Figueiredo C, Takahashi H, Wrasse C M, Otsuka Y, Shiokawa K, Barros D (2018) Medium-scale traveling ionospheric disturbances observed by detrended total electron content maps over Brazil. J Geophys Res Space Phys 123:2215-2227. doi.org/10.1002/2017JA025021

Frissell, N.A., Baker, J., Ruohoniemi, J.M., Gerrard, A.J., Miller, E.S., Marini, J.P., West, M.L., Bristow, W.A., 2014. Climatology of medium-scale traveling ionospheric disturbances observed by the midlatitude blackstone superdarn radar. J. Geophys. Res. Space Phys. 119 (9), 7679-7697, URL:

https://agupubs.onlinelibrary.wiley.com/doi/abs/10.1002/2014JA019870

Fukushima D, Shiokawa K, Otsuka Y, Ogawa T (2012) Observation of equatorial nighttime medium-Scale TID in 630nm airglow images over 7 years. J Geophys Res 117: A10324

Garcia F J, Kelley M C, Makela J J, Huang C S (2000) Airglow observations of mesoscale low-velocity traveling traveling ionospheric disturbances at mid-latitudes, J. Geophys. Res., 105, 18407-18415

Golyandina N, Nekrutkin V, Zhigljavsky A A (2001) Analysis of time series structure: SSA and related techniques. Chapman and Hall, New York

Grant, W B, Pierce R B, Oltmans S J and Browell E V (1998) Seasonal evolution of total and gravity waves-induced laminae in ozonesonde data in the tropics and subtropics. Geophys. Res. Lett. 25, 1863-6

Grocott A, Hosokawa K, Ishida T, Lester M, Milan S E, Freeman M P, Sato N, Yukimatu A S (2013) Characteristics of medium-scale traveling ionospheric disturbances observed near the Antarctic Peninsula by HF radar, J. Geophys. Res. Space Physics. doi:10.1002/jgra.50515

Guanyi C, Chen Z, Yi L, Jiaqi Z, Qiong T, Xiang W, Zhengyu Z (2019) A statistical analysis of medium-scale traveling ionospheric disturbances during 2014-2017 using the Hong Kong CORS network. doi.org/10.1186/s40623019-1031-9, Earth, Planets and Space

Habarulema J B, Katamzi Z T, McKinnell L A (2013a) Estimating the propagation characteristics of large-scale traveling ionospheric disturbances using ground-based and satellite data. J. Geophys. Res. 118, 7768-7782.

Heisler L H (1963) Observation of moveement of perturbations in the F-region. J. Atmospheric Terrest. Phys

Hernández-Pajares M, Juan J M, Sanz J (2006a) Medium-scale traveling ionospheric disturbances affecting GPS measurements: Spatial and temporal analysis, J. Geophys. Res., 111, A07S11, doi: 10.1029/ 2005JA011474

Hernández-Pajares M, Juan J M, Sanz J, Aragón-Àngel A (2012) Propagation of medium scale traveling ionospheric disturbances at different latitudes and solar cycle conditions, Radio Sci., 47, RS0K05, doi:10.1029/2011RS004951

Hines C O (1960) Internal atmospheric gravity waves at ionospheric heights. Canadian Journal of Physics, pp. 14411481

Hocke K, Schlegel K A (1996) Review of atmospheric gravity waves and travelling ionospheric disturbance: 19821995. Max-Planck -Institute fur Aeronomie, Germany, Ann. Geophysicae, 1996 
Hooke William H (1970) The Ionospheric Response to Internal Gravity Wave. Journal of Geophysical Reserach, Space Physics, VOL. 75, No. 28, October 1.

Hooke William H (1968) Ionospheric irregularities produced by internal atmo-spheric gravity waves. J. Atmos. Terr. Phys., 30, $795-823$

Huang C S, Miller C A, and Kelley M C (1994) Basic properties and gravity wave initiation of the mid-latitude F region instability. Radio Science, 29, 395-405, doi:10.1029/93RS01669

Husin A, Abdullah M, Momani M A (2011) Observation of medium-scale traveling ionospheric disturbances over Peninsular Malaysia based on IPP trajectories. Radio Sci., 46, RS2018. doi:10.1029/2010RS004408.

Hunsucker R D (1982) Atmospheric gravity waves generated in the high-latitude ionosphere: A review, Rev. Geophys., 20, 293- 315, doi:10.1029/RG020i002p00293

Jacobson A R, Carlos R C, Massey R S, Wu G (1995) Observations of traveling ionospheric disturbances with a satellite-beacon radio interferometer: Seasonal and local time behavior. J. Geophys. Res, 100, 1653- 1665

Jonah O F, Kherani E A, De-Paula E R (2016) Observation of TEC perturbation associated with mediumscale traveling ionospheric disturbance and possible seeding mechanism of atmospheric gravity wave at a Brazilian sector. J. Geophys. Res. Space Physics, 121, 2531-2546, doi:10.1002/ 2015JA022273

Langley R (1993) GPS world, 4

Kelley M C, Miller C A, (1997) Electrodynamics of midlatitude spread F3. Electrohydrodynamic waves ? A new look at the role of electric fields in thermospheric wave dynamics. J. Geophys. Res., 102, 11,539-11,547, 1997

Kelley M C and Fukao S (1991) Turbulent upwelling of the mid-latitude ionosphere: 2. Theoretical framework. J. Geophys. Res., 96, 3747-3753

Kherani A, De-Paula E, Olusegun J (2013) Observations and simulations of equinoctial asymmetry during low and high solar activities. Presentation at a Proceeding of the Thirteenth International Congress of the Brazilian Geophysical Society, Rio de Janeiro, Brazil, August 26-29

Klobuchar, J.A., 1996. Ionospheric effects on GPS. In: Parkinson, B.W., Spilker, J.J. (Eds.), Global Positioning System: Theory and Application, vol. 1. American Institute of Aeronautics and Astronautics Inc.

Kotake N, Otsuka Y, Ogawa T, Tsugawa T, Saito A (2007) Statistical study of medium-scale traveling ionospheric disturbances observed with the GPS networks in Southern California. Earth Planets and Space 59:95-102. https://doi.org/10.1186/BF03352681

Mannucci A, Wilson B, Yuan D, Ho C, Lindqwister U, Runge T (1998) Radio science, 33, 565

Norsuzila Y, Abdullah M., Ismail M., Zaharim A (2009) Model validation for total electron content (TEC) at an equatorial region Eur. J. Sci. Res. 28 (4), 642-648.

Ogawa T, Igarashi K, Aikyo K, Maeno H (1987) NNSS Satellite observations of medium-scale traveling ionospheric disturbances at southern high-latitudes. J. Geomagn. Geoelec., 39(12), 709-721

Oinats A V, Nishitani N, Ponomarenko P (2016) Statistical characteristics of medium-scale traveling ionospheric disturbances revealed from the Hokkaido East and Ekaterinburg HF radar data. Earth Planet and Space doi.org/10.1186/s40623-016-0390-8

Oluwadare T S, Thai C N, Akala A O, Heise S, Alizadeh, M, Schuh H (2018) Characterization of GPS-TEC over African equatorial ionization anomaly (EIA) region during 2009-2016. Advances in Space Research. doi.org/10.1016/j.asr.2018.08.044. 
Otsuka Y, Onoma F, Shiokawa K, Ogawa T, Yamamoto M, Fukao S (2007) Simultaneous observations of nighttime medium-scale traveling ionospheric disturbances and E region field-aligned irregularities at mid-latitude. J. Geophys. Res., 112, A06317, doi:10.1029/2005JA011548

Otsuka Y, Suzuki K, Nakagawa S, Nishioka M, Shiokawa K, Tsugawa T (2013) GPS observations of medium-scale traveling ionospheric disturbances over Europe, Ann. Geophys., 31, 163-172

Perkins F (1973) Spread F and ionospheric currents. J. Geophys. Res., 78, 218 - 226, doi: 10.1029/JA078i001p00218

Warnant R, Pottiaux E (2000) The increase of the ionospheric activity as measured by GPS. Earth Planets Space, 52, $1055-1060,2000$

Warnant R (1998) Detection of irregularities in the total Electron content using GPS measurements - Application to a mid-latitude station. Acta Geod. Geo1'h. Hung., Vol. 33(1), 1'1'. 121-128, 1998

Samuel H F (1974) A Theory of Medium-Scale Traveling Ionospheric Disturbances. Journal of Geophysical Research vol. 79 , No. 34

Tariku Y A (2015) TEC prediction performance of the IRI-2012 model over Ethiopia during the rising phase of solar cycle 24 (2009-2011). Earth Planet Sp 67, 140 (2015).

Thome G D (1964) Incoherent scatter observations of traveling ionospheric disturbance. J. Geophys. Res. 69, 4047 4049

Tsuda T, Shepherd M, Gopalswamy N (2015) Advancing the understanding of the Sun-Earth interaction-the Climate and Weather of the Sun-Earth System (CAWSES) II program. Prog. in Earth and Planet. Sci. 2, 28.

Tsuda T (2014) Characteristics of atmospheric gravity waves observed using the MU (Middle and Upper atmosphere) radar and GPS (Global Positioning System) radio occultation. Proc Jpn Acad Ser B Phys Biol Sci.;90 (1):12-27. doi:10.2183/pjab.90.12

Tsugawa T, Otsuka Y, Coster A J, Saito A (2007) Medium-scale traveling ionospheric disturbances detected with dense and wide TEC maps over North America. Geophys. Res. Lett., 34, L22101, doi:10.1029/2007GL031663

Tsugawa T, Kotake N, Otsuka Y, Saito A (2006a), Medium-scale traveling ionospheric disturbances observed by GPS receiver network in Japan: A short review, GPS Solutions, 11, 139- 144, doi:10.1007/s10291-006-0045-5.

Valladares C E, Hei M A (2012) Measurement of the characteristics of TID susing small and regional networks of GPS receivers during the campaign of 17-30 July of 2008. International Journal of Geophysics, 2012, 1-14, 2012

Wanninger L (2004), Ionospheric disturbance indices for RTK and network RTK positioning, paper presented at ION GPS, Inst. of Navig., Long Beach, Calif.

Wang L, Alexander J (2009) Gravity wave activity during stratospheric sudden warming in the 2007-2008 Northern Hemisphere winter. J. Geophys. Res., 114, doi:10.1029/2009JD011867

Wang M, Ding F, Wan W (2007) Monitoring global traveling ionospheric disturbances using the worldwide GPS network during the October 2003 storms. Earth Planet Space. doi.org/10.1186/BF03352702

Yeh K C, Liu C H (1969) Theory of Ionospheric Waves, Department of Electrical Engineering, University of Illinois at Urbana-Champaign Urbana. Illinois, Academic Press New York and London

Yokoyama T, Hysell D L (2010) A new midlatitude ionosphere electrodynamics coupling model (MIECO): Latitudinal dependence and propagation of medium-scale traveling ionospheric disturbances. Geophys. Res. Lett., 37 , L08105, doi:10.1029/2010GL042598 
Zhao B, Wan W, Liu L, Ren Z (2009) Characteristics of the ionospheric total electron content of the equatorial ionization anomaly in the Asian-Australian region during 1996-2004. Ann. Geophys. 27, 3861-3873. 